hep-th/0511165

DESY 05-231

ZMP-HH/05-21

\title{
On the infinite-dimensional spin-2 symmetries in Kaluza-Klein theories
}

\author{
Olaf Hohm \\ II. Institut für Theoretische Physik \\ Universität Hamburg \\ Luruper Chaussee 149 \\ D-22761 Hamburg, Germany \\ and \\ Zentrum für Mathematische Physik \\ Universität Hamburg, Bundesstrasse 55 \\ D-20146 Hamburg, Germany \\ email: olaf.hohm@desy.de
}

\begin{abstract}
We consider the couplings of an infinite number of spin-2 fields to gravity appearing in Kaluza-Klein theories. They are constructed as the broken phase of a massless theory possessing an infinite-dimensional spin-2 symmetry. Focusing on a circle compactification of four-dimensional gravity we show that the resulting gravity/spin-2 system in $D=3$ has in its unbroken phase an interpretation as a Chern-Simons theory of the Kac-Moody algebra iso(1,2) associated to the Poincaré group and also fits into the geometrical framework of algebra-valued differential geometry developed by Wald. Assigning all degrees of freedom to scalar fields, the matter couplings in the unbroken phase are determined, and it is shown that their global symmetry algebra contains the Virasoro algebra together with an enhancement of the Ehlers group $S L(2, \mathbb{R})$ to its affine extension. The broken phase is then constructed by gauging a subgroup of the global symmetries. It is shown that metric, spin-2 fields and Kaluza-Klein vectors combine into a Chern-Simons theory for an extended algebra, in which the affine Poincaré subalgebra acquires a central extension.
\end{abstract}

November 2005 


\section{Introduction}

The AdS/CFT correspondence is still one of the most promising avenues towards a deeper understanding of the strong coupling mysteries of gauge theories on the one hand and nonperturbative effects of string or gravity theories on the other hand [1, 2, 3]. However, to use the AdS/CFT correspondence even at the level where the supergravity approximation is valid, one has to compare the full non-linear couplings of in general massive KaluzaKlein states to operators of corresponding conformal dimension on the CFT side, and a restriction to massless modes is therefore unsatisfactory. Moreover, as the internal manifolds appearing in the background geometries of 10-dimensional supergravity, like $A d S_{5} \times S^{5}$ or $A d S_{3} \times S^{3} \times T^{4}$, are typically of the same size as the Anti-de Sitter space, the masses of the higher Kaluza-Klein states are very small, and it is therefore in no sense an effective description to truncate them out. Nevertheless, usually one indeed inspects only the lowest multiplet, simply because of our lack of knowledge concerning the effective actions of the higher ones. This paper aims to go a first step towards a construction of the full action containing the whole tower of Kaluza-Klein modes.

In practice it is a very tedious task even to compute the effective action for the zeromodes. Thus, in the case of a generic internal manifold one would have no chance to compute the action for the full tower of Kaluza-Klein modes directly. Instead one therefore may construct the action directly in the lower-dimensional space-time by identifying the underlying symmetries and deriving the possible couplings which are allowed by them. This approach has been proven to be very fruitful in the construction of gauged supergravities. For instance, in case that the number of unbroken supercharges is sufficiently large it is usually sufficient to identify the underlying gauge group, which is given by the isometry group of the internal manifold, and the spectrum of the various fields under this gauge group. More recently this program has been applied to gauged supergravity in $D=3$ [4, 5]. In this way a tower of massive spin-1 Kaluza-Klein multiplets for supergravity on $A d S_{3} \times S^{3} \times K\left(K=T^{4}\right.$ or $\left.K 3\right)$ has been described as a unique gauging of three-dimensional supergravity [5], where the vectors get massive via a three-dimensional version of the Higgs mechanism. What was missing in this description is the full tower of massive spin-2 multiplets (originating from the higher-dimensional metric and its superpartners). It is our aim in this paper to incorporate towers of massive spin-2-fields via coupling massless spin-2 fields to gravity and establishing a novel version of the Higgs mechanism by which they can become massive.

However, it is not clear a priori whether such a massless spin-2 theory actually exists. Even though we know that the massive theory appearing via Kaluza-Klein reduction has to be consistent, the direct construction of gravity/spin-2 coupled systems seems to be disabled by the so-called interaction problem for higher-spin fields. The latter states that it is not clear how to construct an interacting theory of higher spin fields (with spin $s>1$ ), in particular how to couple them to gravity (for a recent review see [6].) The latter holds for massive and more severely also for massless fields. Let us illustrate this problem in the case of a massless spin-2 field. The action for a free massless field of arbitrary spin on a Minkowski background ${ }^{1}$ has been constructed by Pauli and Fierz [7]

\footnotetext{
${ }^{1}$ Even though we are ultimately interested in the AdS case, we will restrict the analysis to Minkowski backgrounds and discuss later on possible generalizations to AdS spaces.
} 
and is given in the case of a spin-2 field $h_{\mu \nu}$ by

$$
S=\int d^{D} x\left[\frac{1}{2} \partial_{\mu} h_{\nu \rho} \partial^{\mu} h^{\nu \rho}-\partial_{\mu} h^{\mu \nu} \partial^{\rho} h_{\rho \nu}+\partial_{\mu} h^{\mu \nu} \partial_{\nu} \hat{h}-\frac{1}{2} \partial_{\mu} \hat{h} \partial^{\mu} \hat{h}\right],
$$

where $\hat{h}=\eta^{\mu \nu} h_{\mu \nu}$ denotes the trace evaluated in the Minkowski metric. This of course coincides with the linearization of the Einstein-Hilbert action and is moreover invariant under the spin-2 gauge transformations $\delta h_{\mu \nu}=\partial_{\mu} \xi_{\nu}+\partial_{\nu} \xi_{\mu}$, which are the linearized diffeomorphisms. To couple this spin-2 field to gravity, naively one would replace all partial derivatives in (1.1) by covariant ones. However, this would violate the invariance under any obvious covariantisation of the spin-2 transformation due to the non-commutativity of covariant derivatives, $\left[\nabla_{\mu}, \nabla_{\nu}\right] \sim R_{\mu \nu}$. This in turn would render the theory inconsistent since there is no longer a symmetry which reduces the propagating degrees of freedom to the required $\frac{1}{2} D(D-3)$ of spin-2. And indeed it has been proven several times in the literature that spin-2 fields cannot be coupled consistently to gravity (see e.g. [8, 9, 10]). Even though in [11, 12] Cutler and Wald were able to construct interacting multi-graviton theories that are consistent in the sense that they admit a spin-2 gauge invariance, later on it has been shown [9] that all of these theories are unphysical due to the presence of ghost-like excitations.

The reasoning given for the interaction problem so far seems to apply only for massless fields, in which case one has to have a gauge symmetry, but it is actually more severe in that also a coupling of massive higher spin fields to gravity is in general not consistent. (For the case of massive spin-2 fields a very clear discussion of the consistency problems can be found in [10], see also [13] and [14, 15].)

To make this point more transparent, we remind the reader that actually the same problem appears for massless spin-3/2 fields $\psi_{\mu}$, whose free gauge symmetry $\delta \psi_{\mu}=\partial_{\mu} \epsilon$ is also not consistent with gravity couplings. But here it has been partially solved by the invention of local supersymmetry. Therefore a number of massless spin-3/2 fields can be coupled to gravity if they are realized as gauge fields of supersymmetry, and moreover, couplings of massive spin-3/2 fields are only consistent if they are realized through spontaneously broken supersymmetry. The massive spin- $3 / 2$ fields appearing in Kaluza-Klein reductions therefore have to be accompanied by spontaneously broken supercharges. For instance, recently we have constructed the effective supergravity action for a massive spin-3/2 multiplet appearing in the KK reduction on $A d S_{3} \times S^{3} \times S^{3}$ as a spontaneously broken maximal $N=16$ supergravity theory [16], which therefore in total has twice as much supersymmetry than is actually preserved by the background. But the theory admits no symmetric phase, such that half of the gravitinos get massive through a super-Higgs mechanism. However, the possible number of spin-3/2 fields is limited by the maximal amount of 32 real supercharges, and it remains the puzzle how an infinite KK tower of spin-3/2 fields can be coupled to gravity.

One of the main aims of the present paper is to analyze in which way consistency of the gravity/spin-2 coupling in Kaluza-Klein theories can be achieved. That means, we will show in which way a spin-2 symmetry is realized in the full non-linear theory that guarantees consistent couplings in the same sense as supersymmetry does for spin-3/2 fields. More precisely, we will construct an unbroken phase with couplings of an infinite number of massless spin-2 fields to gravity and possessing a larger symmetry structure. This theory will then be deformed via gauging certain rigid symmetries, which in turn 
modifies the spin-2 symmetry. This is in analogy to the deformation of an ungauged into a gauged supergravity, which also modifies the supersymmetry variations.

In this paper we will focus on a KK reduction to $D=3$. This is mainly motivated by the topological nature of massless spin-2 fields and the fact that the analogous construction of gauged supergravities in $D=3$ is significantly simplified due to the fact that in the ungauged theory all propagating bosonic degrees of freedom can be assigned to scalar fields by use of standard Poincaré duality [17, 18. In fact, starting from a formulation where all vectors have been dualized into scalars, the global symmetry group is enhanced, e.g. in the maximally supersymmetric case it is the exceptional group $E_{8(8)}$. Gauging of a subgroup of the global symmetry can in turn be performed by just introducing a topological Chern-Simons term for the gauge fields (and adding some Yukawa-type couplings). The analysis of the possible gaugings consistent with supersymmetry can then be performed in a completely algebraic way. Fortunately, also gauged supergravities with Yang-Mills type gauging are covered by these theories, since the on-shell duality between vectors and scalars actually persists also in the gauged phase, as has been shown in [4, 19].

It will be our aim in this paper to outline a similar procedure for the construction of the effective action of massive KK states in $D=3$, i.e. we have to show that all degrees of freedom can be dualized into scalar fields. In an unbroken limit, where the KK masses go to zero, these will then exhibit an enhanced global symmetry, which severely restricts the form of the couplings. In addition we will have a local spin-2 symmetry for infinitely many massless spin-2 fields in much the same way as one has local supersymmetry in an ungauged supergravity theory. The broken phase of the KK theory will then be constructed by gauging part of the global symmetry, or in other words by switching on a gauge coupling, which will turn out to be given by the mass scale $M$ characterizing the inverse radius of the internal manifold. This gauged deformation in turn will induce a mass term for the spin-2 fields such that a novel Higgs mechanism for the spin-2 fields is possible in the same way as a super-Higgs effect appears in gauged supergravity.

More specifically, we have to address the following questions:

(i) How can we identify the unbroken phase, and how is the spin-2 symmetry realized in this limit? In particular, how does this theory fit into the no-go results discussed in the literature before?

(ii) Which global symmetry is realized on the scalar fields in this phase?

(iii) Which subgroup of the global symmetries has to be gauged in order to get the full Kaluza-Klein theory? Does a formulation exist also for the gauged phase, where all vector and spin-2 fields appear to be topological?

(iv) How does the spin-2 symmetry get modified due to the gauging?

The first step will therefore be to identify the ungauged theory with its symmetries, i.e. to answer question (i). The required symmetries that appear in Kaluza-Klein reductions have in part been analyzed some time ago by Dolan and Duff in [20], where they showed that in the simplest case of an $S^{1}$ compactification including all massive modes a local Virasoro algebra $\hat{v}$ corresponding to the diffeomorphisms on $S^{1}$ as well as the affine extension $\widehat{i s o(1,2)}$ of the Poincaré algebra appear. The latter describes an infinitedimensional spin-2 symmetry, which in the Kaluza-Klein theory is spontaneously broken 
to the usual diffeomorphism group such that all higher spin-2 fields get massive via eating vectors and scalars. We will construct a consistent infinite-dimensional gauge theory for massless spin-2 fields as a Chern-Simons theory of $i \widehat{s o(1,2)}$ and moreover show that

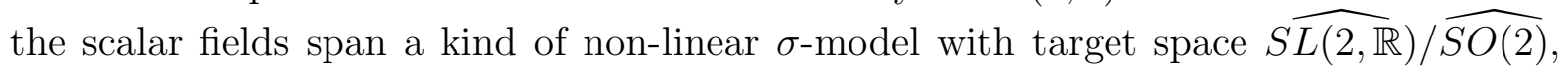
exhibiting an enlarged global symmetry, which answers question (ii). Answering question (iii) we will gauge part of the global symmetry, which in turn modifies the spin-2 symmetries. In the formulation, where all degrees of freedom appear in scalar fields, the KK vectors are topological and we will show that they combine with the spin-2 fields into a Chern-Simons theory for an extended algebra, which enlightens the modification of the spin-2 symmetries, thus answering in part question (iv). Schematically, the situation is given below.

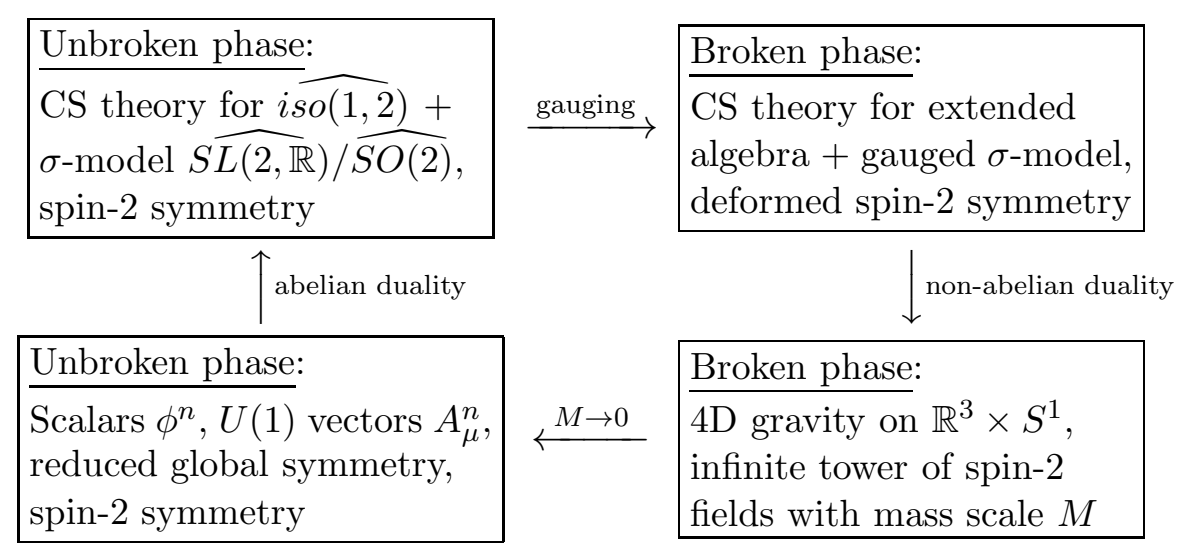

In order to get the full Kaluza-Klein theory (given in the lower right corner) we show how to construct the ungauged theory with its enlarged symmetry group (given in the upper left corner). Then we argue that via gauging part of the global symmetries we obtain a theory (given in the upper right corner) which is on-shell equivalent to the original theory. However, in the case of a reduction on $S^{1}$ the effective action can also be computed directly (and in fact has been computed for reductions to $D=4$, see [21, 22, 23, 24, 25]), i.e. the unbroken phase could then be determined by taking the limit $M \rightarrow 0$ (i.e. obtaining the theory in the lower left corner) and then performing a standard dualization. Nevertheless this is the simplest case, where one can analyze the general aspects of the program outlined above, which is crucial in order to apply this procedure to more complicated internal manifolds.

The paper is organized as follows. After reviewing in sec. 2 the results of [20] applied to the case of an $S^{1}$ compactification to $D=3$, the unbroken phase with vanishing $\mathrm{KK}$ masses will be discussed in sec. 3. For this we will construct a consistent coupling of infinitely many massless spin-2 fields to gravity and discuss its relation to a geometrical framework introduced by Wald in [12. Finally, after dualization we analyze the symmetries of the $\sigma$-model, which is spanned by the scalar fields. In sec. 4 we will turn to the general problem of gauging a subgroup of the global symmetries found in sec. 3 and see in particular how the topological fields combine into an extended Chern-Simons theory, somehow unifying the internal symmetries with the spin-2 symmetries. We conclude in sec. 5 with a discussion, including those aspects which apply also to KK reductions to dimensions other than $D=3$, while a generalization to arbitrary internal manifolds will be discussed in the appendix. 


\section{Kac-Moody symmetries for a Kaluza-Klein theory on $\mathbb{R}^{3} \times S^{1}$}

It has been shown by Dolan and Duff [20] that Kaluza-Klein compactification can be analyzed from the following point of view. The infinite tower of massive modes in the lower-dimensional Kaluza-Klein spectrum can be viewed as resulting from a spontaneous symmetry breaking of an infinite-dimensional Kac-Moody-like algebra down to the Poincaré group times the isometry group of the internal manifold. This infinite dimensional symmetry group is a remnant of the higher dimensional diffeomorphism group.

To be more specific let us review Dolan and Duff's analysis applied to the case of a Kaluza-Klein reduction on $\mathbb{R}^{3} \times S^{1}$. We start from pure Einstein gravity in $D=4$ and split the vielbein $E_{M}^{A}$ in $D=4$ as follows ${ }^{2}$ :

$$
E_{M}^{A}=\left(\begin{array}{cc}
\phi^{-1 / 2} e_{\mu}^{a} & \phi^{1 / 2} A_{\mu} \\
0 & \phi^{1 / 2}
\end{array}\right)
$$

Here we have chosen a triangular gauge and also performed a Weyl rescaling. The fields are now expanded in spherical harmonics of the compact manifold, which for $S^{1}$ simply reads

$$
\begin{aligned}
e_{\mu}^{a}(x, \theta) & =\sum_{n=-\infty}^{\infty} e_{\mu}^{a(n)}(x) e^{i n \theta}, \quad A_{\mu}(x, \theta)=\sum_{n=-\infty}^{\infty} A_{\mu}^{n}(x) e^{i n \theta} \\
\phi(x, \theta) & =\sum_{n=-\infty}^{\infty} \phi^{n}(x) e^{i n \theta}
\end{aligned}
$$

where we have to impose the reality constraint $\left(\phi^{*}\right)^{n}=\phi^{-n}$ and similarly for the other fields. Truncating to the zero-modes, the effective Lagrangian is given by

$$
\mathcal{L}=-e R^{(3)}+\frac{1}{2} e g^{\mu \nu} \phi^{-2} \partial_{\mu} \phi \partial_{\nu} \phi-\frac{1}{4} e \phi^{2} g^{\mu \rho} g^{\nu \sigma} F_{\mu \nu} F_{\rho \sigma}
$$

where as usual $F_{\mu \nu}$ denotes the $U(1)$ field strength for $A_{\mu}$. This action is invariant under three-dimensional diffeomorphisms and $U(1)$ gauge transformations.

Let us next analyze how the four-dimensional symmetries are present in the KaluzaKlein theory without any truncation. For this we notice that the diffeomorphisms in $D=4$, which are locally generated by a vector field $\xi^{M}$, are restricted by the topology of the assumed ground state $\mathbb{R}^{3} \times S^{1}$ to be periodic in $\theta$. Therefore we have to expand similarly

$$
\xi^{\mu}(x, \theta)=\sum_{n=-\infty}^{\infty} \xi^{\mu(n)}(x) e^{i n \theta}, \quad \xi^{5}(x, \theta)=\sum_{n=-\infty}^{\infty} \xi^{5(n)}(x) e^{i n \theta} .
$$

The four-dimensional diffeomorphisms act on the vielbein as

$$
\delta_{\xi} E_{M}^{A}=\xi^{N} \partial_{N} E_{M}^{A}+\partial_{M} \xi^{N} E_{N}^{A}
$$

\footnotetext{
${ }^{2} M, N, \ldots=0,1,2,5$ denote $D=4$ space-time indices, $A, B, \ldots$ are flat $D=4$ indices and the coordinates are called $x^{M}=\left(x^{\mu}, \theta / M\right)$, where $M$ is a mass scale characterizing the inverse radius of the compact dimension. Our metric convention is $(+,-,-)$ for $D=3$ and similar for $D=4$.
} 
and by applying this formula to (2.1) we get

$$
\begin{aligned}
\delta_{\xi} \phi & =\xi^{\rho} \partial_{\rho} \phi+\xi^{5} \partial_{5} \phi+2 \phi \partial_{5} \xi^{\rho} A_{\rho}+2 \phi \partial_{5} \xi^{5} \\
\delta_{\xi} A_{\mu} & =\xi^{\rho} \partial_{\rho} A_{\mu}+\xi^{5} \partial_{5} A_{\mu}+\partial_{\mu} \xi^{\rho} A_{\rho}+\partial_{\mu} \xi^{5}-A_{\mu} \partial_{5} \xi^{\rho} A_{\rho}-A_{\mu} \partial_{5} \xi^{5} \\
\delta_{\xi} e_{\mu}^{a} & =\xi^{\rho} \partial_{\rho} e_{\mu}^{a}+\xi^{5} \partial_{5} e_{\mu}^{a}+\partial_{\mu} \xi^{\rho} e_{\rho}^{a}+\partial_{5} \xi^{5} e_{\mu}^{a}+\partial_{5} \xi^{\rho} A_{\rho} e_{\mu}^{a} .
\end{aligned}
$$

Moreover we have to add a compensating Lorentz transformation with parameter $\tau_{5}^{a}=$ $-\phi^{-1} \partial_{5} \xi^{\rho} e_{\rho}^{a}$ to restore the triangular gauge,

$$
\delta_{\tau} \phi=0, \quad \delta_{\tau} e_{\mu}^{a}=-A_{\mu} \partial_{5} \xi^{\rho} e_{\rho}^{a}, \quad \delta_{\tau} A_{\mu}=-\phi^{-2} \partial_{5} \xi^{\rho} g_{\rho \mu},
$$

where as usual we have written $g_{\mu \nu}=e_{\mu}^{a} e_{\nu a}$, but now with $\theta$-dependent vielbein. Using the mode expansion for the fields in (2.2) and the transformation parameter in (2.4), one gets an infinite-dimensional Kaluza-Klein symmetry acting on the fields $\mathrm{as}^{3}$

$$
\begin{aligned}
\delta \phi^{n} & =\xi_{k}^{\rho} \partial_{\rho} \phi^{n-k}+i M \sum_{k}(n+k) \xi_{k}^{5} \phi^{n-k}+2 i M \sum_{k, l} k \xi_{k}^{\rho} \phi^{n-k-l} A_{\rho}^{l} \\
\delta A_{\mu}^{n} & =\partial_{\mu} \xi_{n}^{5}+i M \sum_{k}(n-2 k) \xi_{k}^{5} A_{\mu}^{n-k}+\xi_{k}^{\rho} \partial_{\rho} A_{\mu}^{n-k}+\partial_{\mu} \xi_{k}^{\rho} A_{\rho}^{n-k} \\
& -i M \sum_{k, l} k \xi_{k}^{\rho}\left(\phi^{-2}\right)^{n-k-l} g_{\rho \mu}^{l}-i M \sum_{k, l} k \xi_{k}^{\rho} A_{\mu}^{n-k-l} A_{\rho}^{l} \\
\delta e_{\mu}^{a(n)} & =\xi_{k}^{\rho} \partial_{\rho} e_{\mu}^{a(n-k)}+\partial_{\mu} \xi_{k}^{\rho} e_{\rho}^{a(n-k)} \\
& +i M n \xi_{k}^{5} e_{\mu}^{a(n-k)}+i M \sum_{k, l} k \xi_{k}^{\rho}\left(e_{\mu}^{a(n-k-l)} A_{\rho}^{l}-e_{\rho}^{a(n-k-l)} A_{\mu}^{l}\right)
\end{aligned}
$$

Here $\left(\phi^{-2}\right)^{n}$ is implicitly defined by $\phi^{-2}=\sum_{n=-\infty}^{\infty}\left(\phi^{-2}\right)^{n} e^{i n \theta}$.

We see now that the standard Kaluza-Klein vacuum given by the vacuum expectation values

$$
\left\langle g_{\mu \nu}\right\rangle=\eta_{\mu \nu}, \quad\left\langle A_{\mu}\right\rangle=0, \quad\langle\phi\rangle=1,
$$

is only invariant under rigid $k=0$ transformations, or in other words the infinitedimensional symmetry is spontaneously broken to the symmetry of the zero-modes, i.e. to three-dimensional diffeomorphisms and a $U(1)$ gauge symmetry.

To explore the group structure which is realized on the whole tower of Kaluza-Klein modes including its spontaneously broken part, Dolan and Duff proceeded as follows. Expanding the generators of the $D=3$ Poincaré algebra as well as those for the diffeomorphisms on $S^{1}$ into Fourier modes, one gets

$$
P_{a}^{n}=e^{i n \theta} \partial_{a}, \quad J_{a b}^{n}=e^{i n \theta}\left(x_{b} \partial_{a}-x_{a} \partial_{b}\right), \quad Q^{n}=-M e^{i n \theta} \partial_{\theta},
$$

where we have used flat Minkowski indices. This implies after introducing $J^{a}=\frac{1}{2} \varepsilon^{a b c} J_{b c}$ the following symmetry algebra

$$
\begin{aligned}
& {\left[P_{a}^{m}, P_{b}^{n}\right]=0, \quad\left[J_{a}^{m}, J_{b}^{n}\right]=\varepsilon_{a b c} J^{c(m+n)}, \quad\left[J_{a}^{m}, P_{b}^{n}\right]=\varepsilon_{a b c} P^{c(m+n)},} \\
& {\left[Q^{m}, Q^{n}\right]=i M(m-n) Q^{m+n},} \\
& {\left[Q^{m}, P_{a}^{n}\right]=-i M n P_{a}^{m+n}, \quad\left[Q^{m}, J_{a}^{n}\right]=-i M n J_{a}^{m+n},}
\end{aligned}
$$

\footnotetext{
${ }^{3}$ We will adopt the Einstein convention also for double indices $m, n=-\infty, \ldots, \infty$, but indicate summations explicitly, if the considered indices appear more than twice.
} 
i.e. we get the Kac-Moody algebra associated to the Poincaré group as well as the Virasoro algebra, both without a central extension. More precisely, we have a semi-

direct product of the Virasoro algebra $\hat{v}$ with the affine Poincaré algebra $\widehat{i s o(1,2)}$ in the standard fashion known from the Sugawara construction [26]. This algebra should be realized as a local symmetry. However, this latter statement is a little bit contrived since even the diffeomorphisms (i.e. the $k=0$ transformations) are known not to be realized in general as gauge transformations for a certain Lie algebra, e.g. as gauge transformations for the $P_{a}$. One aim of the present paper is to clarify this question in the case of a KK reduction to $D=3$, and we will see how a modification of (2.11) appears as a proper gauge symmetry.

In summary, from this infinite-dimensional symmetry algebra only iso $(1,2) \times u(1)$ remains unbroken in the Kaluza-Klein vacuum. This in turn implies that the fields $A_{\mu}^{n}$ and $\phi^{n}$ for $n \neq 0$, which correspond to the spontaneously broken generators $\xi_{n}^{\mu}$ and $\xi_{n}^{5}$, can be identified with the Goldstone bosons. They get eaten by the spin-2 fields $e_{\mu}^{a(n)}$, such that the latter become massive. A massless spin-2 field carries no local degrees of freedom in $D=3$, while a massless vector as well as a real scalar each carry one degree of freedom in $D=3$, such that in total the massive spin-2 fields each carry two degrees of freedom, as expected.

\section{Ungauged phase of the Kaluza-Klein theory}

We have seen in the last section that the full tower of Kaluza-Klein modes on $\mathbb{R}^{3} \times S^{1}$ carries a representation of an infinite-dimensional, but spontaneously broken symmetry algebra. As indicated in the introduction we are going to construct the action for this theory by first starting from its unbroken phase, where all spin-2 fields appear massless and then gauging a certain subgroup of the global symmetries. The unbroken phase is given by the limit, where the mass scale $M \rightarrow 0$, or equivalently, where the radius of the compact dimension goes to infinity. In other words, the gauge coupling $g$ will be determined by this mass scale, $g=M$. In 3.1 and 3.2 we discuss the theory describing the pure spin-2 sector. Additional matter couplings will be introduced in 3.3, while possible dualizations are discussed in sec. 3.4.

\subsection{Infinite-dimensional spin-2 theory}

To construct the theory containing infinitely many massless spin-2 fields coupled to gravity, we remember that according to (2.11) it should have an interpretation as a gauge theory of the Kac-Moody algebra iso(1,2). However, as we already indicated in the discussion at the end of the previous section, in general dimensions this is not a helpful statement, because not even pure gravity has an honest interpretation as a Yang-Millslike gauge theory. Fortunately, Witten has shown that in contrast gravity in $D=3$ can be viewed as a gauge theory of the Poincaré algebra iso $(1,2)$, namely as a Chern-Simons theory for this particular non-compact gauge group [27]. Furthermore, the symmetries of general relativity, i.e. the diffeomorphisms, are on-shell realized as the non-abelian gauge transformations. We are going to show that correspondingly the Chern-Simons theory 
of the affine $\widehat{i s o(1,2)}$ describes a consistent coupling of infinitely many spin-2 fields to gravity.

To start with, we recall the Chern-Simons theory for a gauge connection $\mathcal{A}$, which is given by

$$
S_{C S}=\int \operatorname{Tr}\left(\mathcal{A} \wedge d \mathcal{A}+\frac{2}{3} \mathcal{A} \wedge \mathcal{A} \wedge \mathcal{A}\right)
$$

Here the trace refers symbolically to an invariant and non-degenerate quadratic form on the Lie algebra. The invariance of the quadratic form $\langle$,$\rangle then implies that under an$ arbitrary variation one has

$$
\delta S_{C S}=\int\left\langle\delta \mathcal{A}_{\mu}, \mathcal{F}_{\nu \rho}\right\rangle d x^{\mu} \wedge d x^{\nu} \wedge d x^{\rho}
$$

where $\mathcal{F}_{\mu \nu}=\partial_{\mu} \mathcal{A}_{\nu}-\partial_{\nu} \mathcal{A}_{\mu}+\left[\mathcal{A}_{\mu}, \mathcal{A}_{\nu}\right]$ denotes the field strength. In particular, under a gauge transformation $\delta \mathcal{A}_{\mu}=D_{\mu} u$, where $D_{\mu}$ denotes the gauge covariant derivative

$$
D_{\mu} u=\partial_{\mu} u+\left[\mathcal{A}_{\mu}, u\right]
$$

of an infinitesimal transformation parameter $u$, the action is invariant due to the Bianchi identity. In addition, the non-degeneracy of the quadratic form implies the equations of motion $\mathcal{F}_{\mu \nu}=0$.

Therefore, to construct the Chern-Simons theory for $i \widehat{s o(1,2)}$, we have to find such a quadratic form. It turns out that

$$
\left\langle P_{a}^{m}, J_{b}^{n}\right\rangle=\eta_{a b} \delta^{m,-n}, \quad\left\langle P_{a}^{m}, P_{b}^{n}\right\rangle=\left\langle J_{a}^{m}, J_{b}^{n}\right\rangle=0
$$

defines an invariant form since the bilinear expression

$$
W:=\sum_{n=-\infty}^{\infty} P^{a(n)} J_{a}^{(-n)}
$$

commutes with all gauge group generators. For instance,

$$
\left[W, P_{b}^{k}\right]=\varepsilon_{a b c} \sum_{n=-\infty}^{\infty} P^{a(n)} P^{c(k-n)}=0
$$

can be seen by performing an index shift $n \rightarrow n^{\prime}=k-n$, which shows that the sum is symmetric in $a$ and $c .^{4}$

We turn now to the calculation of the action, the equations of motion and the explicit form of the gauge transformations, which is necessary to identify the Kaluza-Klein symmetries and fields. The gauge field takes values in the Kac-Moody algebra, i.e. it can be written as

$$
\mathcal{A}_{\mu}=e_{\mu}^{a(n)} P_{a}^{n}+\omega_{\mu}^{a(n)} J_{a}^{n} .
$$

\footnotetext{
${ }^{4}$ Upon truncating the quadratic form to the zero-modes, this reduces to an invariant form of the Poincaré algebra, which was the one used in 27. to construct the Chern-Simons action describing pure gravity in $D=3$.
} 
Note, that in the description of ordinary Einstein gravity as Chern-Simons theory the gauge field $\omega_{\mu}^{a}$ is interpreted as the spin-connection, which like in the Palatini formulation is determined only by the equations of motion to be the Levi-Civita connection. Here, instead, we have an infinite number of 'connections' and their meaning will be interpreted later.

With the invariant quadratic form defined in (3.4), the action reads

$$
S_{C S}=\int d^{3} x \varepsilon^{\mu \nu \rho} e_{\mu a}^{(n)}\left(\partial_{\nu} \omega_{\rho}^{a(-n)}-\partial_{\rho} \omega_{\nu}^{a(-n)}+\varepsilon^{a b c} \omega_{\nu b}^{(m)} \omega_{\rho c}^{(-n-m)}\right)
$$

If we define 'generalized' curvatures

$$
R^{a(n)}=d \omega^{a(n)}+\varepsilon^{a b c} \omega_{b}^{(m)} \wedge \omega_{c}^{(n-m)},
$$

the action may be written in a more compact form as

$$
S_{C S}=\int e_{a}^{(n)} \wedge R^{a(-n)}
$$

The field equations implying vanishing field strength, $\mathcal{F}_{\mu \nu}=0$, read in the given case

$$
\begin{array}{r}
\partial_{\mu} e_{\nu}^{a(n)}-\partial_{\nu} e_{\mu}^{a(n)}+\varepsilon^{a b c} e_{\mu b}^{(n-m)} \omega_{\nu c}^{(m)}+\varepsilon^{a b c} \omega_{\mu b}^{(n-m)} e_{\nu c}^{(m)}=0, \\
\partial_{\mu} \omega_{\nu}^{a(n)}-\partial_{\nu} \omega_{\mu}^{a(n)}+\varepsilon^{a b c} \omega_{\mu b}^{(n-m)} \omega_{\nu c}^{(m)}=0 .
\end{array}
$$

Due to the mixing of the infinitely many 'spin connections', the torsion defined by $e_{\mu}^{a(0)}$ does no longer vanish by the equations of motion. This in turn implies that it is not transparent which part of the Einstein equation expresses the curvature and which part the energy-momentum tensor for the higher spin-2 fields. We will clarify this point later.

Next we evaluate the explicit form of the gauge transformations. Introducing the algebra-valued transformation parameter $u=\rho^{a(n)} P_{a}^{n}+\tau^{a(n)} J_{a}^{n}$, for the transformations given by $\delta \mathcal{A}_{\mu}=D_{\mu} u$ one finds

$$
\begin{aligned}
& \delta e_{\mu}^{a(n)}=\partial_{\mu} \rho^{a(n)}+\varepsilon^{a b c} e_{\mu b}^{(n-m)} \tau_{c}^{(m)}+\varepsilon^{a b c} \omega_{\mu b}^{(n-m)} \rho_{c}^{(m)}, \\
& \delta \omega_{\mu}^{a(n)}=\partial_{\mu} \tau^{a(n)}+\varepsilon^{a b c} \omega_{\mu b}^{(n-m)} \tau_{c}^{(m)} .
\end{aligned}
$$

To see that these gauge transformations indeed include the spin-2 Kaluza-Klein transformations (2.8) for $M=0$, let us define for given KK transformation parameterized by $\xi_{k}^{\mu}$ the gauge parameters

$$
\rho^{a(n)}=\xi_{k}^{\mu} e_{\mu}^{a(n-k)}, \quad \tau^{a(n)}=\xi_{k}^{\mu} \omega_{\mu}^{a(n-k)} .
$$

Then the gauge transformation (3.12) takes the form

$$
\begin{aligned}
\delta e_{\mu}^{a(n)} & =\partial_{\mu} \xi_{k}^{\rho} e_{\rho}^{a(n-k)}+\xi_{k}^{\rho} \partial_{\rho} e_{\mu}^{a(n-k)} \\
& +\xi_{k}^{\rho}\left(\partial_{\mu} e_{\rho}^{a(n-k)}-\partial_{\rho} e_{\mu}^{a(n-k)}+\varepsilon^{a b c} e_{\mu b}^{(n-k-m)} \omega_{\rho c}^{(m)}+\varepsilon^{a b c} \omega_{\mu b}^{(n-k-m)} e_{\rho c}^{(m)}\right)
\end{aligned}
$$


where we have again performed an index shift. We see that the first term reproduces the correct KK transformation in (2.8) with $M=0$, while the last term vanishes by the equations of motion (3.11). On-shell the KK transformations are therefore realized as gauge transformations. That the symmetry is realized only on-shell should not come as a surprise because this is already the case for the diffeomorphisms [27, which are now part of the KK-symmetries.

Thus we have determined a theory which is by construction a consistent coupling of infinitely many spin-2 fields. One might ask the question whether the theory can be consistently truncated to a finite number of spin-2 fields, i.e. where only $e_{\mu}^{a(n)}, n=$ $-N, \ldots, N$ for any finite $N$ remain. This turns out not to be the case, because setting all generators of the Kac-Moody algebra with $|n|>N$ to zero does not result in a consistent Lie algebra since the Jacobi identity would be violated, ${ }^{5}$ and correspondingly the Chern-Simons theory would be inconsistent.

So far we have seen that the action permits a consistent spin-2 invariance. It remains to be checked that it can also be viewed as a deformation of a sum of free Pauli-Fierz Lagrangians (1.1), in particular that the first-order theory constructed here is equivalent to a second order action. To see this we introduce an expansion parameter $\kappa$ and linearize the theory by writing

$$
e_{\mu}^{a(0)}=\delta_{\mu}^{a}+\kappa h_{\mu}^{a(0)}+O\left(\kappa^{2}\right), \quad e_{\mu}^{a( \pm 1)}=\kappa h_{\mu}^{a( \pm 1)}+O\left(\kappa^{2}\right) .
$$

We concentrate for simplicity reasons only on the case where just $e_{\mu}^{a( \pm 1)}$ are present. Even though, as we have just seen, this is not consistent with the gauge symmetry in general, it yields correct results up to order $O(\kappa)$ as the corrections by the full equations are at least of order $O\left(\kappa^{2}\right)$. Using the equations of motion for $e_{\mu}^{a( \pm 1)}$ we can now express $\omega_{\mu}^{a( \pm 1)}$ in terms of them. One finds upon expanding up to $O(\kappa)$

$$
\begin{aligned}
\omega_{\mu}^{a(1)}= & \kappa\left[\varepsilon^{\nu a c}\left(\partial_{\mu} h_{\nu c}^{(1)}-\partial_{\nu} h_{\mu c}^{(1)}\right)+\delta_{b}^{\nu}\left(h_{\mu}^{b(1)} \omega_{\nu}^{a(0)}-h_{\mu}^{a(1)} \omega_{\nu}^{b(0)}\right.\right. \\
& \left.+\omega_{\mu}^{b(0)} h_{\nu}^{a(1)}-\omega_{\mu}^{a(0)} h_{\nu}^{b(1)}\right)-\frac{1}{4} \varepsilon^{\sigma \rho d}\left(\partial_{\rho} h_{\sigma d}^{(1)}-\partial_{\sigma} h_{\rho d}^{(1)}\right) \delta_{\mu}^{a} \\
& \left.-\frac{1}{4} \delta^{\rho c} \delta^{\sigma d}\left(h_{\rho d}^{(1)} \omega_{\sigma c}^{(0)}-h_{\rho c}^{(1)} \omega_{\sigma d}^{(0)}+\omega_{\rho d}^{(0)} h_{\sigma c}^{(1)}-\omega_{\rho d}^{(0)} h_{\sigma c}^{(1)}\right) \delta_{\mu}^{a}\right]+O\left(\kappa^{2}\right),
\end{aligned}
$$

and analogously for $\omega_{\mu}^{a(-1)}$. The next step would be to insert these relations into the equation for $e_{\mu}^{a(0)}$ and solve the resulting expression for the 'spin connection' $\omega_{\mu}^{a(0)}$. However, due to the fact that $e_{\mu}^{a( \pm 1)}$ as well as $\omega_{\mu}^{a( \pm 1)}$ are of order $O(\kappa)$, for the approximation linear in $\kappa$ we just get

$$
0=\varepsilon^{a b c} \delta_{\mu b} \omega_{\nu c}^{(0)}+\varepsilon^{a b c} \delta_{\nu c} \omega_{\mu b}^{(0)}+\kappa\left(\partial_{\mu} h_{\nu}^{a(0)}-\partial_{\nu} h_{\mu}^{a(0)}+\varepsilon^{a b c}\left(h_{\mu b}^{(0)} \omega_{\nu c}^{(0)}+\omega_{\mu b}^{(0)} h_{\nu c}^{(0)}\right)\right) .
$$

In the limit $\kappa \rightarrow 0$ this is the relation for vanishing torsion in the flat case and is therefore solved by $\omega_{\mu}^{a(0)}=0$. Up to order $O(\kappa)$ we have the usual relation of vanishing torsion for the 'metric' $h_{\mu}^{a(0)}$ and it can therefore be solved as in the standard case. But to solve the

\footnotetext{
${ }^{5}$ Similarly it has been shown in 28 , that a restriction to a finite number of massive spin-2 fields in Kaluza-Klein theories is not a consistent truncation.
} 
equation we have to multiply with the inverse vielbein and therefore up to order $O(\kappa)$ the solution is just the linearized Levi-Civita connection. Altogether we have

$$
\omega_{\mu}^{a(0)}=\kappa(\text { lin. Levi-Civita connection })+O\left(\kappa^{2}\right) \text {. }
$$

We can now insert this relation into the formulas (3.16) for $\omega_{\mu}^{a( \pm 1)}$ and get

$$
\omega_{\mu}^{a(1)}=\kappa\left[\varepsilon^{\nu a c}\left(\partial_{\mu} h_{\nu c}^{(1)}-\partial_{\nu} h_{\mu c}^{(1)}\right)-\frac{1}{4} \varepsilon^{\sigma \rho d}\left(\partial_{\rho} h_{\sigma d}^{(1)}-\partial_{\sigma} h_{\rho d}^{(1)}\right) \delta_{\mu}^{a}\right]+O\left(\kappa^{2}\right)
$$

and analogously for $\omega_{\mu}^{a(-1)}$. Finally inserting this expression into the equation of motion for $\omega_{\mu}^{a(1)}$ to that order, i.e.

$$
0=\partial_{\mu} \omega_{\nu}^{a(1)}-\partial_{\nu} \omega_{\mu}^{a(1)}
$$

and multiplying with $\varepsilon^{\mu \nu \lambda}$ results in

$$
0=\partial_{\mu} \partial^{\lambda} h^{\mu \nu}-\partial^{\nu} \partial^{\lambda} \hat{h}+\eta^{\lambda \nu} \square \hat{h}-\eta^{\lambda \nu} \partial_{\mu} \partial_{\rho} h^{\mu \rho}+\partial^{\nu} \partial_{\mu} h^{\mu \lambda}-\square h^{\nu \lambda} .
$$

This coincides exactly with the equation of motion derived from the original free spin-2 action (1.1). Here we have defined $h_{\mu \nu}:=\delta_{\mu}^{a} h_{\nu a}^{(1)}+\delta_{\nu}^{a} h_{\mu a}^{(1)}$, which also results up to $O(\kappa)$ from the general formula for the higher spin-2 fields in a metric-like representation:

$$
g_{\mu \nu}^{(n)}:=e_{\mu}^{a(n-m)} e_{\nu a}^{(m)} .
$$

Clearly, also for the linearized Einstein equation we get the free spin-2 equation, and therefore we can summarize our analysis by saying that the theory reduces in the linearization up to order $O(\kappa)$ to a sum of Pauli-Fierz terms. On the other hand, we know that the full theory has the KK symmetries (2.8) which mix fields of different level and accordingly the full theory (3.8) has to include non-linear couplings. This in turn implies that the higher order terms in $\kappa$ cannot vanish and our theory is therefore a true deformation of a pure sum of Pauli-Fierz terms. In summary, one can solve the equations of motion for $\omega_{\mu}^{a(n)}$ at least perturbatively, thus giving a second-order formulation. However, it would be much more convenient to have a deeper geometrical understanding for the $\omega_{\mu}^{a(n)}$. Such a geometrical interpretation indeed exists and is given by Wald's algebravalued differential geometry [12, which we are going to discuss in the next section.

\subsection{Geometrical interpretation of the spin-2 symmetry}

Cutler and Wald analyzed in [11] the question of possible consistent extensions of a free spin-2 gauge invariance to a collection of spin-2 fields. In much the same way as a nonabelian Lie algebra determines the gauge symmetry for a collection of spin-1 fields, they found that such a spin-2 theory is organized by an associative and commutative algebra $\mathfrak{A}$ (which should not be confused with a Lie algebra). Namely, the additional index which indicates the different spin-2 fields is to be interpreted as an algebra index, and therefore any collection of spin-2 fields can be viewed as a single spin-2 field, which takes values in a nontrivial algebra. Now, an associative and commutative algebra $\mathfrak{A}$ can be characterized by its multiplication law, which is with respect to a basis given by a tensor $a_{n m}^{k}$ according to

$$
(v \cdot w)^{n}=a_{m k}^{n} v^{m} w^{k}
$$


where $v, w \in \mathfrak{A}$. That the algebra is commutative and associative is encoded in the relations

$$
a_{m n}^{k}=a_{(m n)}^{k}, \quad a_{m n}^{k} a_{l p}^{n}=a_{n p}^{k} a_{m l}^{n} .
$$

With respect to such a given algebra $\mathfrak{A}$, the allowed gauge transformations can be written according to [11] as

$$
\delta g_{\mu \nu}^{(n)}=\partial_{(\mu} \xi_{\nu)}^{(n)}-2 \Gamma_{\mu \nu}^{\sigma n}{ }_{l} \xi_{\sigma}^{(l)}=: \nabla_{\mu} \xi_{\nu}^{(n)}+\nabla_{\nu} \xi_{\mu}^{(n)}
$$

where the generalized Christoffel symbol is defined by

$$
\Gamma_{\mu \nu l}^{\sigma n}=\frac{1}{2} g^{\sigma \rho k}\left(\partial_{\mu} g_{\rho \nu k}^{n}+\partial_{\nu} g_{\rho \mu k}^{n}-\partial_{\rho} g_{\mu \nu k}^{n}\right)
$$

and

$$
g_{\mu \nu n}^{k}=a_{n m}^{k} g_{\mu \nu}^{(m)}
$$

We see that $\nabla_{\mu}$ has the formal character of a covariant derivative.

Moreover, it has been shown in [12] that beyond this formal resemblance to an ordinary metric-induced connection, there exists a geometrical interpretation in the following sense. As in pure general relativity, where the symmetry transformations are given by the diffeomorphisms acting on the fields via the pullback, the above given transformation rules are the infinitesimal version of a diffeomorphism on a generalized manifold. This new type of manifold introduced in [12] generalizes the notion of an ordinary real manifold to 'algebra-valued' manifolds, where the algebra $\mathfrak{A}$ replaces the role of $\mathbb{R}$. To be more precise, such a manifold is locally modeled by a $n$-fold cartesian product $\mathfrak{A}^{n}$ in the same sense as an ordinary manifold is locally given by $\mathbb{R}^{n}$. On these manifolds one can correspondingly define a metric which looks from the point of view of the underlying real manifold like an ordinary, but algebra-valued metric. Now, the diffeomorphisms of these generalized manifolds act infinitesimally on the metric exactly as written above. (For further details see [12.) Moreover, most of the constructions known from Riemannian geometry like the curvature tensor have their analogue here.

To check whether our theory fits into this general framework we first have to identify the underlying commutative algebra. Due to the fact that the theory contains necessarily an infinite number of spin-2 fields, the algebra has to be infinite-dimensional, too, and we will assume that the formalism applies also to this case.

We will argue that the algebra is given by the algebra of smooth functions on $S^{1}$, on which we had compactified, together with the point-wise multiplication of functions as the algebra structure. ${ }^{6}$ With respect to the complete basis $\left\{e^{i n \theta}, n=-\infty, \ldots, \infty\right\}$ of functions on $S^{1}$, the multiplication is given due to elementary Fourier analysis by

$$
(f \cdot g)^{n}=\sum_{m=-\infty}^{\infty} f^{n-m} \cdot g^{m}=\sum_{k, m=-\infty}^{\infty} \delta_{k+m, n} f^{k} g^{m},
$$

\footnotetext{
${ }^{6}$ That the spin- 2 couplings arising in Kaluza-Klein compactifications might be related to Wald's framework in this way has first been suggested by Reuter in [29, where he analyzed the reduction of a dimensionally continued Euler form in $D=6$. Namely, the latter has the exceptional property of inducing an infinite tower of massless spin-2 fields due to the existence of an infinite-dimensional symmetry already in the higher-dimensional theory.
} 
such that the algebra is characterized by

$$
a_{k m}^{n}=\delta_{k+m, n}
$$

This implies that the metric can be written according to (3.27) as

$$
g_{\mu \nu k}^{n}=a_{k m}^{n} g_{\mu \nu}^{(m)}=g_{\mu \nu}^{(n-k)} .
$$

Now it can be easily checked that the KK transformations (2.8) for $M=0$ applied to (3.22) can be written as

$$
\delta g_{\mu \nu}^{(n)}=\nabla_{\mu} \xi_{\nu}^{(n)}+\nabla_{\nu} \xi_{\mu}^{(n)}
$$

i.e. they have exactly the required form. Here the connection $\nabla_{\mu}$ is calculated as in (3.25) with respect to the algebra (3.29). For this we have assumed that indices are raised and lowered according to

$$
\xi_{\mu}^{(n)}=g_{\mu \nu k}^{n} \xi^{(k) \nu}
$$

while the inverse metric is defined through the relation

$$
g_{k}^{\mu \rho{ }_{k \nu m}} \underset{\rho \nu}{k}=\delta_{\nu}^{\mu} \delta_{m}^{n}
$$

With the help of this geometrical interpretation we are now also able to interpret the existence of an infinite number of 'spin-connections' $\omega_{\mu}^{a(n)}$. If we assume that the vielbeins are invertible in the sense of (3.33), one can solve the equations of motion (3.11) for the connections in terms of $e_{\mu}^{a(n)}$, as we have argued in sec. 3.1. Then one can define a generalized covariant derivative by postulating the vielbein to be covariantly constant,

$$
\nabla_{\mu} e_{\nu}^{a(n)}=\partial_{\mu} e_{\nu}^{a(n)}-\Gamma_{\mu \nu m}^{\rho n} e_{\rho}^{a(m)}+\omega_{\mu b}^{a(n-m)} e_{\nu}^{b(m)}=0
$$

Since the antisymmetric part $\nabla_{[\mu} e_{\nu]}^{a(n)}$ vanishes already by the equations of motion (3.11), this requirement specifies the symmetric part of $\nabla_{\mu} e_{\nu}^{a(n)}$. In turn, the algebra-valued metric (3.22) is covariantly constant with respect to this symmetric connection,

$$
\nabla_{\mu} g_{\nu \rho}^{(n)}=\partial_{\mu} g_{\nu \rho}^{(n)}-\Gamma_{\mu \nu m}^{\sigma n} g_{\sigma \rho}^{(m)}-\Gamma_{\mu \rho}^{\sigma n}{ }_{m} g_{\sigma \nu}^{(m)}=0
$$

But this is on the other hand also the condition which uniquely fixes the Christoffel connection in (3.26) as a function of the algebra-valued metric [12. Altogether, the equations of motion for the Chern-Simons action (3.8) together with (3.34) determine a symmetric connection, which is equivalent to the algebra-valued Christoffel connection (3.26) compatible with the metric (3.22).

Wald also constructed an algebra-valued generalization of the Einstein-Hilbert action, whose relation to the Chern-Simons action (3.8) we are going to discuss now. This generalization is (written for three space-times dimensions) given by

$$
S^{m}=\int a_{n l}^{m} R^{n} \varepsilon_{\mu \nu \rho}{ }^{l} d x^{\mu} \wedge d x^{\nu} \wedge d x^{\rho},
$$


where $R^{n}$ and $\varepsilon_{\mu \nu \rho}^{l}$ denote the algebra-valued scalar curvature and volume form, respectively [12]. Applied to the algebra (3.29) it yields

$$
S^{m}=\int R^{m-n} \varepsilon_{\mu \nu \rho}^{n} d x^{\mu} \wedge d x^{\nu} \wedge d x^{\rho}
$$

Here the volume form is given by $\varepsilon_{\mu \nu \rho}{ }^{n}=e^{n} \epsilon_{\mu \nu \rho}$, where

$$
e^{n}=\frac{1}{3 !} \epsilon^{\mu \nu \rho} \epsilon_{a b c} e_{\mu}^{a(n-m-k)} e_{\nu}^{b(m)} e_{\rho}^{c(k)}
$$

such that the zero-component of (3.37) indeed coincides with the Chern-Simons action (3.10). One may wonder about the meaning of the other components of the algebravalued action (3.37), whose equations of motion cannot be neglected for generic algebras. However, the quadratic form (3.4) which has been used to construct the Chern-Simons action (3.8), is actually not unique, but instead there is an infinite series of quadratic forms,

$$
\left\langle P_{a}^{m}, J_{b}^{n}\right\rangle_{k}=\eta_{a b} \delta^{m, k-n}
$$

each of which is invariant and can therefore be used to define a Chern-Simons action. These will then be identical to the corresponding components of the algebra-valued action (3.37). But, as all of these actions imply the same equations of motion, namely $\mathcal{F}_{\mu \nu}=0$, and are separately invariant under gauge transformations, there is no need to consider the full algebra-valued metric, but instead the zero-component is sufficient.

Moreover, also matter couplings can be described in this framework in a spin-2covariant way. For instance, an algebra-valued scalar field $\phi^{n}$ can be coupled via

$$
S_{\mathrm{scalar}}^{m}=\int a_{n k}^{m} a_{l p}^{n} \partial_{\lambda} \phi^{l} \partial^{\lambda} \phi^{p} \varepsilon_{\mu \nu \rho}^{k} d x^{\mu} \wedge d x^{\nu} \wedge d x^{\rho}
$$

Furthermore, (3.40) is invariant under algebra-diffeomorphisms. The latter acts as a Lie derivative on algebra-valued tensor fields [12, such that the scalars transform with respect to the algebra (3.29) exactly as required by (2.8) in the phase $M \rightarrow 0$. Again, for the algebra (3.29) considered here, the zero-component of (3.40) is separately invariant and can be written as

$$
S_{\text {scalar }}=\int d^{3} x \sqrt{g}^{-n} \partial_{\mu} \phi^{l} \partial^{\mu} \phi^{n-l}=\int d^{3} x d \theta \sqrt{g} \partial_{\mu} \phi \partial^{\mu} \phi .
$$

Similarly, the Chern-Simons action can be rewritten by retaining a formal $\theta$-integration and assuming all fields to be $\theta$-dependent. For explicit computations it is accordingly often more convenient to work with $\theta$-dependent expressions and therefore we will give subsequent formulas in both versions.

Finally let us briefly discuss the resolution of the aforementioned no-go theorems for consistent gravity/spin-2 couplings. In [9] it has been shown that Wald's algebravalued spin-2 theories for arbitrary algebras generically contain ghost-like excitations. Namely, the algebra has to admit a metric specifying the kinetic Pauli-Fierz terms in the free-field limit and moreover has to be symmetric in the sense that lowering the upper index in $a_{m n}^{k}$ by use of this metric results in a totally symmetric $a_{m n k}=a_{(m n k)}$. Now, 
requiring the absence of ghosts, i.e. assuming the metric to be positive-definite, restricts the algebra to a direct sum of one-dimensional ideals (which means $a_{m n}^{k}=0$ whenever $m \neq n)$. The theory reduces in turn to a sum of independent Einstein-Hilbert terms. For the infinite-dimensional algebra considered here the metric is given by the $L^{2}$-norm for square-integrable functions (see formula (A.2) in the appendix), which is clearly positivedefinite. The action may instead be viewed as an integral over Einstein-Hilbert terms and is thus in agreement with 9 .

\subsection{Non-linear sigma-model and its global symmetries}

Apart from the spin-2 sector also the infinite tower of scalar fields $\phi^{n}$ will survive in the unbroken limit $M \rightarrow 0$. We have seen in the last section how spin-2 invariant couplings for scalar fields can be constructed. To fix the actual form of these couplings, we have to identify also the global symmetries in this limit, and in order to uncover the maximal global symmetry, we will dualize all degrees of freedom into scalars.

We note from (2.8) that in the unbroken phase the Virasoro algebra $\hat{v}$ parameterized by $\xi_{k}^{5}$ reduces to an abelian gauge symmetry, while the full $\hat{v}$ will then turn out to be realized only as a global symmetry, which is typical for an ungauged limit. More precisely, we expect an invariance under rigid transformations of the general form

$$
\delta_{\xi^{5}} \chi^{n}=i \sum_{k}(n-(1-\Delta) k) \xi_{k}^{5} \chi^{n-k}
$$

where $\xi_{k}^{5}$ is now space-time independent. One easily checks that these are representations of $\hat{v}$, which can therefore be labeled by their conformal dimension $\Delta$. More precisely, the KK fields $e_{\mu}^{a}, A_{\mu}$ and $\phi$ transform as $\Delta=1, \Delta=-1$ and $\Delta=2$, respectively.

We start form the zero-mode action (2.3) and replace it by the algebra-valued generalization discussed in the last section. For the Einstein-Hilbert term we have already seen that this procedure yields the correct $i \widehat{s o(1,2)}$ gauge theory, and therefore it is sufficient to focus on the scalar kinetic term and the Yang-Mills term. The action reads

$$
S_{\text {matter }}=\int d^{3} x d \theta e\left(-\frac{1}{4} \phi^{2} F^{\mu \nu} F_{\mu \nu}+\frac{1}{2} \phi^{-2} g^{\mu \nu} \partial_{\mu} \phi \partial_{\nu} \phi\right)
$$

where all fields are now $\theta$-dependent or, equivalently, algebra-valued.

To dualize the $U(1)$ gauge fields $A_{\mu}^{n}$ into new scalars $\varphi^{n}$, we define the standard duality relation

$$
\phi^{2} F_{\mu \nu}=e \varepsilon_{\mu \nu \rho} g^{\rho \sigma} \partial_{\sigma} \varphi
$$

which is not affected by the $\theta$-dependence of all fields. Thus, the abelian duality between vectors and scalars persists also in the algebra-valued case, and the degrees of freedom can be assigned to $\phi$ and $\varphi$. The Lagrangian for the scalar fields then takes in the unbroken limit $M \rightarrow 0$ the form

$$
\mathcal{L}_{\text {scalar }}=\frac{1}{2} e g^{\mu \nu} \phi^{-2}\left(\partial_{\mu} \phi \partial_{\nu} \phi+\partial_{\mu} \varphi \partial_{\nu} \varphi\right)
$$


which coincides formally with the zero-mode action after a standard dualization, but now with all fields still being $\theta$-dependent. From (3.44) one determines the transformation properties of the dual scalar $\varphi$ under $\hat{v}$ and finds

$$
\delta_{\xi^{5}} \varphi=\xi^{5} \partial_{5} \varphi+2 \varphi \partial_{5} \xi^{5}
$$

i.e. it transforms in the same representation as $\phi$ with $\Delta=2$. (For the computation it is crucial to take into account that also $e_{\mu}^{a}$ transforms under $\hat{v}$.) Now one easily checks that the action is invariant under global Virasoro transformations.

Moreover, it is well known that the zero-mode scalar fields span a non-linear $\sigma$-model with coset space $S L(2, \mathbb{R}) / S O(2)$ as target space, carrying the 'Ehlers group' $S L(2, \mathbb{R})$ as isometry group [30. If one includes all Kaluza-Klein modes at $M=0$, this symmetry is enhanced to an infinite-dimensional algebra, which we are going to discuss now. Defining the complex scalar field $Z=\varphi+i \phi$, the action can be rewritten as

$$
\mathcal{L}_{\text {scalar }}=\frac{1}{2} e g^{\mu \nu} \frac{\partial_{\mu} Z \partial_{\nu} \bar{Z}}{(Z-\bar{Z})^{2}}
$$

which is invariant under the $S L(2, \mathbb{R})$ isometries acting as

$$
Z \rightarrow Z^{\prime}=\frac{a Z+b}{c Z+d}, \quad\left(\begin{array}{ll}
a & b \\
c & d
\end{array}\right) \in S L(2, \mathbb{R}) .
$$

This invariance is not spoiled by the fact that $Z$ is still $\theta$-dependent and so the $S L(2, \mathbb{R})$ acts on the full tower of Kaluza-Klein modes, as can be seen by expanding (3.48) into Fourier modes. But moreover, also the $S L(2, \mathbb{R})$ group elements can depend on $\theta$, and therefore an additional infinite-dimensional symmetry seems to appear.

To determine the algebra structure of this infinite-dimensional symmetry, let us first introduce a basis for $\operatorname{sl}(2, \mathbb{R})$ :

$$
h=\left(\begin{array}{cc}
1 & 0 \\
0 & -1
\end{array}\right), \quad e=\left(\begin{array}{ll}
0 & 1 \\
0 & 0
\end{array}\right), \quad f=\left(\begin{array}{ll}
0 & 0 \\
1 & 0
\end{array}\right) .
$$

Infinitesimally, with transformation parameter $\alpha=\alpha(\theta)$ they act as

$$
\delta_{\alpha}(h) Z=-2 \alpha Z, \quad \delta_{\alpha}(e) Z=-\alpha, \quad \delta_{\alpha}(f) Z=\alpha Z^{2},
$$

or, expanded in Fourier components, as

$$
\delta_{\alpha^{m}}(h) Z^{n}=-2 \alpha^{m} Z^{n-m}, \quad \delta_{\alpha^{m}}(e) Z^{n}=-\delta^{m n} \alpha^{m}, \quad \delta_{\alpha^{m}}(f) Z^{n}=\alpha^{m} Z^{n-m-l} Z^{l} .
$$

In particular, the real part of $Z$, i.e. the dual scalar $\varphi$, transforms as a shift under $e$ transformations, which will later on be promoted to local shift symmetries in the gauged theory.

We can now compute the closure of these symmetry variations with the Virasoro variations $\delta_{\xi^{m}}(Q)$. One finds

$$
\begin{aligned}
& {\left[\delta_{\xi^{m}}(Q), \delta_{\eta^{n}}(h)\right] Z^{k}=-i n \delta_{(\xi \eta)^{m+n}}(h) Z^{k},} \\
& {\left[\delta_{\xi^{m}}(Q), \delta_{\eta^{n}}(e)\right] Z^{k}=i(-n-2 m) \delta_{(\xi \eta)^{m+n}}(e) Z^{k},} \\
& {\left[\delta_{\xi^{m}}(Q), \delta_{\eta^{n}}(f)\right] Z^{k}=i(-n+2 m) \delta_{(\xi \eta)^{m+n}}(f) Z^{k},}
\end{aligned}
$$


where we have set

$$
(\xi \eta)^{m+n}=\xi^{m} \eta^{n}
$$

Furthermore, the extended $\operatorname{sl}(2, \mathbb{R})$ transformations close among themselves according to

$$
\begin{aligned}
{\left[\delta_{\alpha^{m}}(h), \delta_{\beta^{n}}(e)\right] Z^{k} } & =2 \delta_{(\alpha \beta)^{m+n}}(e) Z^{k} \\
{\left[\delta_{\alpha^{m}}(h), \delta_{\beta^{n}}(f)\right] Z^{k} } & =-2 \delta_{(\alpha \beta)^{m+n}}(f) Z^{k}, \\
{\left[\delta_{\alpha^{m}}(e), \delta_{\beta^{n}}(f)\right] Z^{k} } & =\delta_{(\alpha \beta)^{m+n}}(h) Z^{k}, \\
{\left[\delta_{\alpha^{m}}(h), \delta_{\alpha^{n}}(h)\right] Z^{k} } & =\left[\delta_{\alpha^{m}}(e), \delta_{\alpha^{n}}(e)\right] Z^{k}=\left[\delta_{\alpha^{m}}(f), \delta_{\alpha^{n}}(f)\right] Z^{k}=0 .
\end{aligned}
$$

Altogether we can conclude that the following Lie algebra is a global symmetry of the ungauged theory

$$
\begin{aligned}
{\left[Q^{m}, Q^{n}\right] } & =i(m-n) Q^{m+n}, \quad\left[Q^{m}, e_{n}\right]=i(-n-2 m) e_{m+n}, \\
{\left[Q^{m}, h_{n}\right] } & =-i n h_{m+n}, \quad\left[Q^{m}, f_{n}\right]=i(-n+2 m) f_{m+n}, \\
{\left[h_{m}, e_{n}\right] } & =2 e_{m+n}, \quad\left[h_{m}, f_{n}\right]=-2 f_{n+m}, \\
{\left[e_{m}, f_{n}\right] } & =h_{m+n}, \quad\left[e_{m}, e_{n}\right]=\left[h_{n}, h_{m}\right]=\left[f_{m}, f_{n}\right]=0 .
\end{aligned}
$$

We see that the symmetry algebra includes not only the Virasoro algebra $\hat{v}$, but also the Kac-Moody algebra $\widehat{s l(2, \mathbb{R})}$, which transforms under $\hat{v}$. Note, that these transformation properties are not the standard ones known from the Sugawara construction (compare the form of $\hat{v} \ltimes i \widehat{s o(1,2)}$ in sec. 2, see also [26]). However, this algebra reduces to the standard form upon the change of basis given by $\hat{Q}^{m}=Q^{m}+m h^{m}$, such that it clearly defines a consistent Lie algebra.

In summary, we can think of the scalar fields $\phi^{n}$ and $\varphi^{n}$ as parameterizing an infinitedimensional $\sigma$-model coset space

$$
\mathcal{M}=\frac{\widehat{S L(2, \mathbb{R})}}{\widehat{S O(2)}} .
$$

Strictly speaking this is not full truth, since the metric used to contract indices is actually algebra-valued. Thus, here we have an algebra-valued generalization of a $\sigma$-model, which in turn is the reason that it does not only have the symmetries $s \widehat{l(2, \mathbb{R})}$, but instead the whole algebra $\hat{v} \ltimes s \widehat{l(2, \mathbb{R})}$ defined by $(\underline{3.55})$.

In total, the ungauged phase of the effective Kaluza-Klein action without any truncation is therefore given by

$$
\begin{aligned}
S=\int d^{3} x & \left(-\varepsilon^{\mu \nu \rho} e_{\mu a}^{(n)}\left(\partial_{\nu} \omega_{\rho}^{a(-n)}-\partial_{\rho} \omega_{\nu}^{a(-n)}+\varepsilon^{a b c} \omega_{\nu b}^{(m)} \omega_{\rho c}^{(-n-m)}\right)\right. \\
+ & \left.\frac{1}{2} e g^{\mu \nu} \phi^{-2}\left(\partial_{\mu} \phi \partial_{\nu} \phi+\partial_{\mu} \varphi \partial_{\nu} \varphi\right)\right)
\end{aligned}
$$

where in the second term the algebra multiplication defined in (3.40) is implicit, or in other words, where all fields are $\theta$-dependent and an integration over $\theta$ is assumed. 
The action is by construction invariant under spin-2 transformations. Moreover, we have already seen that the scalar couplings are also invariant under global Virasoro transformations. To see that this is also the case for the generalized Einstein-Hilbert term, we have to show that one can determine the transformation rule for $\omega_{\mu}^{a(n)}$ such that the action stays invariant. This is indeed possible, and one finds

$$
\delta_{\xi^{5}} \omega_{\mu}^{a(n)}=i \sum_{k}(n-k) \xi_{k}^{5} \omega_{\mu}^{a(n-k)}, \quad \delta_{\xi^{5}} \omega_{\mu}^{a}=\xi^{5} \partial_{\theta} \omega_{\mu}^{a}
$$

Equivalently, they can be computed by solving the $\omega_{\mu}^{a(n)}$ in terms of the vielbeins by use of (3.11) and then applying a $\hat{v}$ transformation to this expression. Both results coincide. Note that instead the full algebra-valued action (3.37) transforms non-trivially under $\hat{v}$, namely as

$$
\delta S^{m}=i m \xi_{n}^{5} S^{(m-n)} .
$$

However, as we have already seen in sec. 3.2, it is sufficient to include only the zerocomponent in (3.57), which is clearly invariant.

\subsection{Dualities and gauging}

So far we have determined the unbroken phase of the KK theory in a description where all propagating degrees of freedom reside in scalar fields. Before we turn to a gauging of a subgroup of the global symmetries one may wonder whether it is still possible to assign all degrees of freedom to scalars, since the introduction of gauge fields necessarily seems to enforce the appearance of local degrees of freedom that are instead carried by vectors. However, in [4, 19] it has been shown that in three-dimensional gauged supergravities all Yang-Mills-type gaugings are on-shell equivalent to Chern-Simons gaugings (with an enlarged number of scalar fields), in which case the gauge fields are topological and thus all bosonic degrees of freedom still appear as scalar fields. We are going to show that this duality also applies to the present case.

To begin with, we note that in the gauged theory all partial derivatives are replaced by covariant ones. For a given field $\chi$ transforming in a representation $\Delta$ under $\hat{v}$ the covariant derivative reads

$$
D_{\mu} \chi^{n}=\partial_{\mu} \chi^{n}-i g \sum_{k}(n-(1-\Delta) k) A_{\mu}^{k} \chi^{n-k},
$$

where we have introduced the gauge coupling $g=M$. Indeed, it transforms by construction covariantly under local $\hat{v}$ transformations, $\delta_{\xi}\left(D_{\mu} \chi^{n}\right)=i g(n-(1-\Delta) k) \xi_{k} D_{\mu} \chi^{n-k}$, if we assume as usual that $A_{\mu}^{n}$ transforms as a gauge field under the adjoint (i.e. as the KK vector in (2.8) with $\Delta=-1$ ). Similarly, the non-abelian $\hat{v}$ field strength is given by

$$
F_{\mu \nu}^{n}=\partial_{\mu} A_{\nu}^{n}-\partial_{\nu} A_{\mu}^{n}+i g \sum_{m}(n-2 m) A_{\mu}^{n-m} A_{\nu}^{m} .
$$

These expressions are given for the KK fields in $\theta$-dependent notation by

$$
\begin{aligned}
D_{\mu} \phi & =\partial_{\mu} \phi-g A_{\mu} \partial_{\theta} \phi-2 g \phi \partial_{\theta} A_{\mu}, \\
F_{\mu \nu} & =\partial_{\mu} A_{\nu}-\partial_{\nu} A_{\mu}-g A_{\mu} \partial_{\theta} A_{\nu}+g A_{\nu} \partial_{\theta} A_{\mu} .
\end{aligned}
$$


The part of the gauged action containing scalar fields will be given by the covariantisation of the action (3.43) according to (3.62). One easily checks that this transforms into a total $\theta$-derivative under local $\xi_{k}^{5}$-transformations, i.e. defines an invariant action. Furthermore, by explicit reductions [21, 22] it has been shown that exactly these terms appear, as well as an explicit $\hat{v}$ gauge invariant mass term for the spin-2 fields, i.e. the action reads

$$
\mathcal{L}_{\text {scalar }}=\frac{1}{2} e g^{\mu \nu} \phi^{-2} D_{\mu} \phi D_{\nu} \phi-\frac{1}{4} e \phi^{2} g^{\mu \rho} g^{\nu \sigma} F_{\mu \nu} F_{\rho \sigma}+\mathcal{L}_{\text {mass }}
$$

To show that this action is indeed on-shell equivalent to a Chern-Simons gauged theory we introduce following [4, 19] new gauge fields for each of the former Yang-Mills fields, or in other words, we enhance the gauge symmetry with nilpotent shift symmetries (see also [31]). To explain this dualization procedure, let us consider the Yang-Mills equation resulting from (3.63)

$$
D^{\mu}\left(\phi^{2} F_{\mu \nu}\right)=j_{\nu}
$$

where $j_{\nu}$ denotes the current induced by the charged fields. It implies integrability of the duality relation

$$
\frac{1}{2} e^{-1} \varepsilon^{\mu \nu \rho} \phi^{2} F_{\nu \rho}=D^{\mu} \varphi+g B^{\mu}=: \mathcal{D}^{\mu} \varphi,
$$

where $\varphi$ will be the scalar field carrying the former degrees of freedom of $A_{\mu}$, and $B_{\mu}$ is the gauge field corresponding to the enlargement of the gauge group. From the previous section we know already the transformation properties of $\varphi$ under $\hat{v}$, and one may check explicitly that it does not change in the gauged phase. In particular, also the dual vector $B_{\mu}$ will transform with $\Delta=2$, in other words it transforms under the dual of the adjoint representation of $\hat{v}$, which will later on turn out to be important. ${ }^{7}$ To define the dual action we add instead of the Yang-Mills term a Chern-Simons-like term $B \wedge F$, where $F$ denotes the non-abelian field strength, and get

$$
\mathcal{L}_{\text {scalar }}=\frac{1}{2} e g^{\mu \nu} \phi^{-2}\left(D_{\mu} \phi D_{\nu} \phi+\mathcal{D}_{\mu} \varphi \mathcal{D}_{\nu} \varphi\right)-\frac{1}{2} g \varepsilon^{\mu \nu \rho} B_{\mu} F_{\nu \rho}+\mathcal{L}_{\text {mass }} .
$$

Indeed, varying with respect to $B_{\mu}$ one recovers the duality relation (3.65), and eliminating the dual scalar $\varphi$ by means of this relation yields the Yang-Mills type theory (3.63). Thus we have shown that the degrees of freedom of the $A_{\mu}^{n}$ can be assigned to new scalars $\varphi^{n}$, if at the same time new topological gauge fields $B_{\mu}^{n}$ are introduced that promote the former global shift transformations (i.e. the $e$-transformations of $\widehat{s l(2, \mathbb{R})})$ to a local symmetry.

\footnotetext{
${ }^{7}$ For each representation $\rho$ on a vector space $V$ one has the dual representation $\rho^{*}$ on the dual space $V^{*}$, which is defined by the requirement $\left\langle\rho^{*}(g)\left(v^{*}\right), \rho(g)(v)\right\rangle=\left\langle v^{*}, v\right\rangle$, where $\langle$,$\rangle denotes the natural$ pairing between vectors in $V$ and $V^{*}$ and $g$ is a group element. This implies $\rho^{*}(g)={ }^{t} \rho\left(g^{-1}\right)$ or at the level of the Lie algebra $\rho^{*}(X)=-{ }^{t} \rho(X)$. Since the adjoint representation is given by $\left(t^{n}\right)_{k}^{m}=f_{k}^{n m}$, the co-adjoint has therefore the matrices $\left(t_{n}^{*}\right)_{m}^{k}=-\left(t^{n}\right)_{m}^{k}=-f_{m}^{n k}$.
} 


\section{Gauged phase of the Kaluza-Klein theory}

Up to now we have determined the action (3.57) of the ungauged theory, which is invariant under global $\hat{v} \ltimes s \widehat{l(2, \mathbb{R})}$ transformations as well as local spin-2 transformations. We argued that in order to get the full Kaluza-Klein action one has to gauge the Virasoro algebra together with the shift symmetries of (3.55). In the next section we discuss the effect of this gauging on the topological fields. We will see that they combine into a single Chern-Simons theory, quite analogous to gauged supergravities, where truncating to the topological fields results in the Chern-Simons theories of 32 for $A d S$-supergroups. The scalars will be discussed thereafter.

\subsection{Local Virasoro invariance for topological fields}

As usual the gauging proceeds in several steps. First of all, one has to replace all partial derivatives by covariant ones. Let us start with the generalized Einstein-Hilbert term. The covariant derivative for $\omega_{\mu}^{a(n)}$ in accordance with (3.58) reads

$$
D_{\mu} \omega_{\nu}^{a(n)}=\partial_{\mu} \omega_{\nu}^{a(n)}-i g \sum_{m}(n-m) A_{\mu}^{m} \omega_{\nu}^{a(n-m)}
$$

or equivalently

$$
D_{\mu} \omega_{\nu}^{a}=\partial_{\mu} \omega_{\nu}^{a}-A_{\mu} \partial_{5} \omega_{\nu}^{a} .
$$

The covariantized Einstein-Hilbert action is then invariant under local Virasoro transformations. In contrast it will no longer be invariant under all spin- 2 transformations, but only under three-dimensional diffeomorphisms, since the explicit $\partial_{5}$ appearing in the covariant derivatives will also act on the spin-2 transformation parameter. Thus, the gauging will deform the spin-2 transformations.

Furthermore, we have already seen that in order to guarantee that the resulting action will be equivalent to the original Yang-Mills gauged theory, one has to introduce a Chern-Simons term for the Kaluza-Klein vectors $A_{\mu}^{n}$, whose propagating degrees of freedom are now carried by the dual scalars $\varphi^{n}$, as well as for the dual gauge fields $B_{\mu}^{n}$. This implies that we do not have to gauge only the Virasoro algebra $\hat{v}$, but instead the whole subalgebra of (3.55), which is spanned by $\left(Q^{m}, e_{m}\right)$, while the rigid symmetry given by $h_{m}$ and $f_{m}$ will be broken explicitly. Both gauge fields combine into a gauge field for this larger algebra. Moreover, in contrast to $\hat{v}$ itself this algebra carries a non-degenerate invariant quadratic form, namely

$$
\left\langle Q^{m}, e_{n}\right\rangle=\delta^{n,-m}
$$

such that a Chern-Simons action can be defined. The existence of this form is due to the fact that $e_{m}$ transforms actually under the co-adjoint action of $\hat{v}$, as we have argued in 3.4. We will see that the Chern-Simons action with respect to this quadratic form indeed reproduces the correct $B \wedge F$-term in (3.66).

It is tempting to ask, whether all topological fields, i.e. the gravitational fields together with the gauge fields for the Virasoro and shift symmetry, can be combined into 
a Chern-Simons theory for a larger algebra. The latter would have to combine the affine Poincaré algebra with the algebra spanned by $\left(Q^{m}, e_{m}\right)$. Naively one would think that the semi-direct product $\hat{v} \ltimes i \widehat{s o(1,2)}$ defined in (2.11) and extended by $e_{m}$ according to (3.55) is the correct choice. However, it does not reproduce the right KK symmetry transformations, and moreover, the algebra seems not to admit a non-degenerate and invariant quadratic form. To see that a Chern-Simons formulation nevertheless exists, we observe that varying the total action consisting of the sum of $\hat{v}$-covariantized Einstein-Hilbert action and $B \wedge F$ with respect to $A_{\mu}$, we get the non-abelian field strength for $B_{\mu}$ plus terms of the form $e_{\mu}^{a} \partial_{5} \omega_{\nu a}$. Thus, a Chern-Simons interpretation is only possible if the latter terms are contained in the field strength of $B_{\mu}$, or in other words, if the algebra also closes according to $[P, J] \sim e$.

Demanding consistency with the Jacobi identities and requiring that $e_{\mu}^{a}$ and $\omega_{\mu}^{a}$ transform under the right representation of $\hat{v}$, the following Lie algebra is then uniquely fixed up to a free parameter $\alpha$ :

$$
\begin{aligned}
{\left[P_{a}^{m}, J_{b}^{n}\right] } & =\varepsilon_{a b c} P^{c(m+n)}+i \alpha n \eta_{a b} e_{m+n}, \quad\left[J_{a}^{m}, J_{b}^{n}\right]=\varepsilon_{a b c} J^{c(m+n)}, \\
{\left[P_{a}^{m}, P_{b}^{n}\right] } & =0 \\
{\left[Q^{m}, Q^{n}\right] } & =i g(m-n) Q^{m+n}, \quad\left[Q^{m}, P_{a}^{n}\right]=i g(-m-n) P_{a}^{m+n}, \\
{\left[Q^{m}, J_{a}^{n}\right] } & =-i g n J_{a}^{m+n}, \quad\left[Q^{m}, e_{n}\right]=i g(-n-2 m) e_{m+n}, \\
{\left[P_{a}^{m}, e_{n}\right] } & =\left[J_{a}^{m}, e_{n}\right]=\left[e_{m}, e_{n}\right]=0 .
\end{aligned}
$$

Here we have rescaled the $Q^{m}$ with the gauge coupling constant $g$ for later convenience.

We see that one gets an algebra which looks similar to the one proposed in [20] (see (2.11) ), except that it does not contain the semi-direct product of $\hat{v}$ with the affine Poincaré algebra, since the $P_{a}^{n}$ and $J_{a}^{n}$ transform in different representations of $\hat{v}$. But in contrast to sec. 3.3, where we observed a similar phenomenon for the global symmetry algebra, there seems not to exist an obvious change of basis which reduces the algebra to the standard form. Namely, because of the different index structure the $Q^{m}$ can be shifted neither by $P_{a}^{m}$ nor $J_{a}^{m}$. In fact, that the algebra is consistent even in this non-standard form is possible only because of the nilpotency of translations, i.e. $\left[P_{a}^{m}, P_{b}^{n}\right]=0$.

Furthermore, we observe that the algebra admits a central extension $e_{m}$ of the Poincaré algebra even at the classical level. (Even though, strictly speaking, it is only a central extension for the Poincaré subalgebra, since the $e_{m}$ do not commute with the $Q^{m}$.) Remarkably, it is exactly this modification of the algebra that allows the existence of an invariant quadratic form. Namely, the bilinear expression

$$
W=P^{a(-m)} J_{a}^{(m)}+\frac{\alpha}{g} Q^{m} e_{-m}
$$

(in particular, $\left\langle Q^{m}, e_{n}\right\rangle=\frac{g}{\alpha} \delta^{m,-n}$ ) is invariant under (4.4). The total Chern-Simons action constructed with respect to this quadratic form, with the gauge field written as

$$
\mathcal{A}_{\mu}=e_{\mu}^{a(n)} P_{a}^{n}+\omega_{\mu}^{a(n)} J_{a}^{n}+A_{\mu}^{n} Q^{n}+B_{\mu}^{n} e_{n},
$$

is then indeed given by

$$
S_{C S}=\int d^{3} x d \theta\left(\varepsilon^{\mu \nu \rho} e_{\mu a}\left(D_{\nu} \omega_{\rho}^{a}-D_{\rho} \omega_{\nu}^{a}+\varepsilon^{a b c} \omega_{\nu b} \omega_{\rho c}\right)+\frac{g}{\alpha} \varepsilon^{\mu \nu \rho} B_{\mu} F_{\nu \rho}\right)
$$


i.e. consists of the $\hat{v}$-covariantized Einstein-Hilbert term and the Chern-Simons action for $A_{\mu}$ and $B_{\mu}$.

Let us briefly comment on the reality constraints on (4.4). Naively one would take (4.4) as real Lie algebra, and correspondingly the gauge fields in (4.6) would also be real. However, the reality condition $\left(Q^{*}\right)^{m}=Q^{m}$ (and similarly for all other generators) is not consistent, since taking the complex conjugate of (4.4) changes relative signs. Instead, only the reality constraint $\left(Q^{*}\right)^{m}=Q^{-m}$ can be consistently imposed. This is on the other hand also in accordance with the reality condition for the original KK fields in (2.2), and therefore the fields in (4.6) fulfill exactly the correct reality constraint. For the Virasoro subalgebra this corresponds to a real section where the Möbius group spanned by $Q^{-1}, Q^{0}$ and $Q^{1}$ is not $S L(2, \mathbb{R})$, but instead the compact $S U(2)$.

The equations of motion for the Chern-Simons action in (4.7) again imply vanishing field strength,

$$
\mathcal{F}_{\mu \nu}=R_{\mu \nu}^{a(n)} J_{a}^{n}+T_{\mu \nu}^{a(n)} P_{a}^{n}+F_{\mu \nu}^{n} Q^{n}+G_{\mu \nu}^{n} e_{n}=0
$$

whose components can in turn be written as

$$
\begin{aligned}
R_{\mu \nu}^{a(n)} & =\partial_{\mu} \omega_{\nu}^{a(n)}-\partial_{\nu} \omega_{\mu}^{a(n)}+\varepsilon^{a b c} \omega_{\mu b}^{(n-m)} \omega_{\nu c}^{(m)} \\
& +i g \sum_{m}(n-m) \omega_{\mu}^{a(n-m)} A_{\nu}^{m}-i g \sum_{m} m A_{\mu}^{n-m} \omega_{\nu}^{a(m)}, \\
T_{\mu \nu}^{a(n)} & =D_{\mu} e_{\nu}^{a(n)}-D_{\nu} e_{\mu}^{a(n)}+\varepsilon^{a b c} e_{\mu b}^{(n-m)} \omega_{\nu c}^{(m)}+\varepsilon^{a b c} \omega_{\mu b}^{(n-m)} e_{\nu c}^{(m)}, \\
F_{\mu \nu}^{n} & =\partial_{\mu} A_{\nu}^{n}-\partial_{\nu} A_{\mu}^{n}+i g \sum_{m}(n-2 m) A_{\mu}^{n-m} A_{\nu}^{m}, \\
G_{\mu \nu}^{n} & =\partial_{\mu} B_{\nu}^{n}-\partial_{\nu} B_{\mu}^{n}+i g \sum_{m}(m-2 n) A_{\mu}^{n-m} B_{\nu}^{m}+i g \sum_{m}(n+m) B_{\mu}^{n-m} A_{\nu}^{m} \\
& +i \alpha \sum_{m} m e_{\mu}^{a(n-m)} \omega_{\nu a}^{(m)}-i \alpha \sum_{m}(n-m) \omega_{\mu}^{a(n-m)} e_{\nu a}^{(m)} .
\end{aligned}
$$

Here $D_{\mu} e_{\nu}^{a(n)}$ denotes the $\hat{v}$-covariant derivative on $e_{\mu}^{a(n)}$, which is in $\theta$-notation given by

$$
D_{\mu} e_{\nu}^{a}=\partial_{\mu} e_{\nu}^{a}-A_{\mu} \partial_{5} e_{\nu}^{a}-e_{\nu}^{a} \partial_{5} A_{\mu} .
$$

Moreover, all quantities can be rewritten in $\theta$-dependent notation, e.g. the non-abelian field strength for $B_{\mu}$ is given by

$$
\begin{aligned}
G_{\mu \nu} & =\partial_{\mu} B_{\nu}-\partial_{\nu} B_{\mu}+2\left(B_{\mu} \partial_{5} A_{\nu}-B_{\nu} \partial_{5} A_{\mu}\right)-A_{\mu} \partial_{5} B_{\nu}+A_{\nu} \partial_{5} B_{\mu} \\
& +\alpha\left(e_{\mu}^{a} \partial_{\theta} \omega_{\nu a}-e_{\nu}^{a} \partial_{\theta} \omega_{\mu a}\right) .
\end{aligned}
$$

The gauge transformations for gauge parameter $u=\rho^{a(n)} P_{a}^{n}+\tau^{a(n)} J_{a}^{n}+\xi_{n}^{5} Q^{n}+\Lambda^{n} e_{n}$ 
can be written as

$$
\begin{aligned}
\delta e_{\mu}^{a(n)} & =\partial_{\mu} \rho^{a(n)}+\varepsilon^{a b c} e_{\mu b}^{(n-m)} \tau_{c}^{(m)}+\varepsilon^{a b c} \omega_{\mu b}^{(n-m)} \rho_{c}^{(m)} \\
& -i g n A_{\mu}^{n-m} \rho^{a(m)}+i g n e_{\mu}^{a(n-m)} \xi_{m}^{5} \\
\delta \omega_{\mu}^{a(n)} & =\partial_{\mu} \tau^{a(n)}+\varepsilon^{a b c} \omega_{\mu b}^{(n-m)} \tau_{c}^{(m)} \\
& -i g \sum_{m} m A_{\mu}^{n-m} \tau^{a(m)}+i g \sum_{m}(n-m) \omega_{\mu}^{a(n-m)} \xi_{m}^{5} \\
\delta A_{\mu}^{n} & =\partial_{\mu} \xi_{5}^{n}+i g \sum_{m}(n-2 m) \xi_{m}^{5} A_{\mu}^{n-m}, \\
\delta B_{\mu}^{n} & =\partial_{\mu} \Lambda^{n}+i g \sum_{m}(m-2 n) \Lambda^{m} A_{\mu}^{n-m}+i g \sum_{m}(n+m) \xi_{m}^{5} B_{\mu}^{n-m} \\
& +i \alpha \sum_{m} m e_{\mu}^{a(n-m)} \tau_{a}^{(m)}+i \alpha \sum_{m}(m-n) \omega_{\mu}^{a(n-m)} \rho_{a}^{(m)} .
\end{aligned}
$$

Let us now check, whether the KK symmetries are included in these gauge transformations. First of all, it reproduces the correct transformation rule for $B_{\mu}$ under $\hat{v}$, as can be seen by rewriting the last equation of (4.12) in $\theta$-dependent notation

$$
\delta B_{\mu}=\partial_{\mu} \Lambda-2 g \Lambda \partial_{\theta} A_{\mu}-g A_{\mu} \partial_{\theta} \Lambda+g \xi^{5} \partial_{\theta} B_{\mu}+2 g B_{\mu} \partial_{\theta} \xi^{5}+\alpha e_{\mu}^{a} \partial_{\theta} \tau_{a}-\alpha \rho_{a} \partial_{\theta} \omega_{\mu}^{a} .
$$

By comparing (4.12) with (2.8) we also see that the Virasoro gauge transformations parameterized by $\xi^{5}$ are correctly reproduced for $e_{\mu}^{a}$ and $A_{\mu}$. To compare with the spin-2 transformations we define in analogy to (3.13) the transformation parameter

$$
\rho^{a}=\xi^{\rho} e_{\rho}^{a}, \quad \tau^{a}=\xi^{\rho} \omega_{\rho}^{a}, \quad \xi^{5}=\xi^{\rho} A_{\rho} .
$$

Then one finds for the vielbein

$$
\delta_{\xi} e_{\mu}^{a}=\xi^{\rho} \partial_{\rho} e_{\mu}^{a}+\partial_{\mu} \xi^{\rho} e_{\rho}^{a}+g A_{\rho} \partial_{\theta} \xi^{\rho} e_{\mu}^{a}-g A_{\mu} \partial_{\theta} \xi^{\rho} e_{\rho}^{a}-\xi^{\rho} T_{\rho \mu}^{a},
$$

which implies that on-shell, i.e. for $T_{\mu \nu}^{a}=0$, the gauge transformations coincide with the KK symmetries in (2.6) and (2.7). With the same transformation parameter and $\Lambda=\xi^{\rho} B_{\rho}$ we find for $A_{\mu}$ and $B_{\mu}$ the following transformation rules (again up to field strength terms)

$$
\begin{aligned}
& \delta_{\xi} A_{\mu}=\xi^{\rho} \partial_{\rho} A_{\mu}+\partial_{\mu} \xi^{\rho} A_{\rho}-g A_{\mu} \partial_{\theta} \xi^{\rho} A_{\rho}, \\
& \delta_{\xi} B_{\mu}=\xi^{\rho} \partial_{\rho} B_{\mu}+\partial_{\mu} \xi^{\rho} B_{\rho}-g A_{\mu} \partial_{\theta} \xi^{\rho} B_{\rho}+2 g B_{\mu} \partial_{\theta} \xi^{\rho} A_{\rho}+\alpha e_{\mu}^{a} \partial_{\theta} \xi^{\rho} \omega_{\rho a},
\end{aligned}
$$

which reproduces for $A_{\mu}$ the same transformation as in (2.8), up to the $\phi$-dependent term (which, of course, cannot be contained in a Chern-Simons formulation.)

As in the case of the pure gravity-spin-2 theory, the topological phase of the KaluzaKlein theory is given by a Chern-Simons theory, and moreover the KK symmetry transformations are on-shell equivalent to the non-abelian gauge transformations determined by (4.4). Even though this equivalence holds only on-shell, the KK transformations are separately an (off-shell) symmetry, since $\delta_{\xi} \mathcal{A}_{\mu}=\xi^{\rho} \mathcal{F}_{\rho \mu}$ leaves the Chern-Simons action invariant, as can be easily checked with (3.2). 
Finally, let us check that spin-2 transformations together with the Virasoro transformations build a closed algebra, as it should be at least on-shell, since they were constructed as Yang-Mills gauge transformations. For the vielbein, e.g., one finds ${ }^{8}$

$$
\left[\delta_{\xi}, \delta_{\eta^{5}}\right] e_{\mu}^{a}=\delta_{(\eta \xi)} e_{\mu}^{a}-\delta_{(\xi \eta)^{5}} e_{\mu}^{a},
$$

with the parameter given by

$$
(\eta \xi)^{\rho}=\eta^{5} \partial_{5} \xi^{\rho}, \quad(\xi \eta)^{5}=\xi^{\rho} \partial_{\rho} \eta^{5}
$$

The same formula holds for $A_{\mu}$ and $B_{\mu}$. But, for $B_{\mu}$ one also has to check the closure of the shift symmetries with spin-2 and here one finds

$$
\left[\delta_{\xi}, \delta_{\Lambda}\right] B_{\mu}=-\delta_{\tilde{\Lambda}} B_{\mu}-2 \Lambda \partial_{5} \xi^{\rho} F_{\rho \mu}
$$

where

$$
\tilde{\Lambda}=\xi^{\rho} \partial_{\rho} \Lambda+2 \Lambda \partial_{5} \xi^{\rho} A_{\rho}
$$

Therefore the algebra closes only on-shell, i.e. if $F_{\mu \nu}=0$.

\subsection{Virasoro-covariantisation for the scalars}

To summarize the results of the last section, we have seen that in the gauged phase the spin-2 transformations of sec. 3 are no longer a symmetry due to the substitution of partial derivatives by covariant ones. Therefore the spin- 2 transformations have to be deformed by $g$-dependent terms. For the topological fields we have seen that a ChernSimons formulation exists, which in turn yields modified spin-2 transformations, which are consistent by construction.

Next let us focus on the scalar fields. For them we have already noted the form of the covariant derivative in (3.62), and the same formula holds for $\varphi$, but with the difference that it also has to be covariant with respect to the local shift symmetries gauged by $B_{\mu}$. The latter act as $\delta_{\Lambda} \varphi=-g \Lambda$, i.e. the covariant derivative reads in $\theta$-notation

$$
\mathcal{D}_{\mu} \varphi=\partial_{\mu} \varphi-A_{\mu} \partial_{5} \varphi-2 \varphi \partial_{5} A_{\mu}+g B_{\mu}
$$

Altogether, replacing the partial derivatives in (3.57) by covariant ones and adding the Chern-Simons action constructed in the last section as well as an explicit mass term which is known to appear [22, 24], results in

$$
\begin{aligned}
S_{\mathrm{KK}}=\int d^{3} x d \theta\left[\varepsilon^{\mu \nu \rho}(\right. & \left.-e_{\mu}^{a}\left(D_{\nu} \omega_{\rho a}-D_{\rho} \omega_{\nu a}+\varepsilon_{a b c} \omega_{\nu}^{b} \omega_{\rho}^{c}\right)-\frac{1}{2} g B_{\mu} F_{\nu \rho}\right) \\
+ & \left.\frac{1}{2} e g^{\mu \nu} \phi^{-2}\left(D_{\mu} \phi D_{\nu} \phi+\mathcal{D}_{\mu} \varphi \mathcal{D}_{\nu} \varphi\right)+\mathcal{L}_{\text {mass }}\right]
\end{aligned}
$$

Here we have determined the free parameter of the algebra (4.4) to be $\alpha=2$ in order to get the correct Chern-Simons term for $A_{\mu}$ and $B_{\mu}$ discussed in sec. 3.4. Namely, there

\footnotetext{
${ }^{8}$ As before, we indicate Virasoro transformations by a subscript 5 on the transformation parameter.
} 
we have already observed that varying this action with respect to $B_{\mu}$ one recovers the duality relation (3.65). In turn, the equations of motion for (4.22) and for the Yang-Mills gauged action are equivalent. This can be seen directly by imposing the gauge $\varphi=0$ in (4.22) and then integrating out $B_{\mu}$, which results exactly in the Kaluza-Klein action containing the Yang-Mills term in (3.63). Moreover, varying with respect to $\omega_{\mu}^{a}$ still implies $T_{\mu \nu}^{a}=0$. This shows that $\omega_{\mu}^{a}$ can be expressed in terms of $e_{\mu}^{a}$ as is standard, but with the exception that all derivatives on $e_{\mu}^{a}$ are now $\hat{v}$-covariant. In the second-order formulation this means that the Einstein-Hilbert part looks formally the same as in sec. 3 , but with all Christoffel symbols now containing $\hat{v}$-covariant derivatives. This is on the other hand also what one gets by direct Kaluza-Klein reduction in second-order form [22, 24. Thus we have shown, that (4.22) is on-shell equivalent to the Kaluza-Klein action which results from dimensional reduction.

In view of the fact that (4.22) is manifestly $\hat{v}$ and shift invariant it remains the question how the spin-2 symmetries are realized. As for the case of the topological fields, also the $\sigma$-model action for the scalar fields will no longer be invariant under the unmodified spin-2 transformations for the same reasons. To find the deformed transformation rule for the scalars, one way is to check the closure of the algebra. The unmodified spin-2 transformations do not build a closed algebra with the local $\hat{v}$ transformations. But, if we deform the spin-2 transformation to

$$
\delta_{\xi} \phi=\xi^{\rho} \partial_{\rho} \phi+2 g \phi \partial_{\theta} \xi^{\rho} A_{\rho}
$$

the algebra closes according to

$$
\left[\delta_{\xi}, \delta_{\eta^{5}}\right] \phi=\delta_{(\eta \xi)} \phi-\delta_{(\xi \eta)^{5}} \phi
$$

i.e. exactly like in the case of the topological fields with the parameters given in (4.18). The KK transformations can therefore be entirely reconstructed by requiring closure of the algebra. The same transformation holds for the dual scalar $\varphi$.

In the presence of matter fields we have to be careful about the closure of the algebra also on the gauge fields. Namely, for the pure Chern-Simons theory shift with spin-2 transformations in (4.20) close on-shell (as it should), but for the theory constructed here the field strength does not vanish. Thus one way to get a closing algebra is to extend the transformation rule according to

$$
\delta^{\prime} B_{\mu}=-2 \varphi \partial_{\theta} \xi^{\rho} F_{\mu \rho}
$$

and all transformations close off-shell.

Therefore we see that in the full theory the transformation rules for the vectors $A_{\mu}$ and $B_{\mu}$ get extended by scalar field dependent terms. That is on the other hand also what we already know from the symmetry variations in (2.8) for $A_{\mu}^{n}$, and these terms will be needed in order for the full action to be spin-2 invariant. This is in complete analogy to the construction of gauged supergravities, where the procedure of gauging is only consistent with supersymmetry, if additional couplings like mass terms are added, while the supersymmetry variations are supplemented by scalar-dependent terms. However, in the present case the invariance of the Yang-Mills gauged Kaluza-Klein theory is guaranteed by construction, which in turn implies that the on-shell equivalent dual theory (4.22) is also invariant (if one assumes transformation rules for $B_{\mu}$, which are on-shell given 
by the variation of the left-hand side of (3.65). In view of our aim to construct the Kaluza-Klein theories for more general backgrounds, it would however be important to find a systematic procedure to determine the scalar-dependent corrections for gaugings of arbitrary diffeomorphism Lie algebras. This we will leave for future work, but here let us just show how the scalar-dependent correction in (4.25) ensures the invariance under spin-2 for a subsector.

For this it will be convenient to separate from the spin-2 transformations those parts which represent already a symmetry for each term separately. To do so we remember that to realize the spin-2 transformations on the topological fields as gauge transformations we had to switch on also the Virasoro transformations with parameter $\xi^{5}=\xi^{\rho} A_{\rho}$. Now we will turn the logic around and apply a spin-2 transformation followed by a Virasoro transformation with parameter $\xi^{5}=-\xi^{\rho} A_{\rho}$. Since Virasoro invariance is manifest, this is a symmetry if and only if spin-2 is a symmetry. One may easily check that on $e_{\mu}^{a}$ and $\phi$ (as well as $\varphi$ ) this transformation is given by

$$
\begin{aligned}
\delta_{\xi} \phi & =\xi^{\rho} D_{\rho} \phi \\
\delta_{\xi} e_{\mu}^{a} & =\xi^{\rho} D_{\rho} e_{\mu}^{a}+D_{\mu} \xi^{\rho} e_{\rho}^{a} .
\end{aligned}
$$

Here we have used (4.10) and also introduced a Virasoro covariant derivative for the spin-2 transformation parameter (of which we may think as transforming as $\delta_{\eta^{5}} \xi^{\mu}=\eta^{5} \partial_{5} \xi^{\mu}$ ),

$$
D_{\mu} \xi^{\rho}=\partial_{\mu} \xi^{\rho}-A_{\mu} \partial_{5} \xi^{\rho}
$$

We see that we get transformation rules which look formally like a diffeomorphism symmetry, except that all appearing derivatives are $\hat{v}$-covariant. In the following we will refer to these transformations as 'gauged diffeomorphisms'. In contrast, the gauge fields $A_{\mu}$ and $B_{\mu}$ transform as

$$
\begin{aligned}
& \delta_{\xi} A_{\mu}=\xi^{\rho} F_{\rho \mu} \\
& \delta_{\xi} B_{\mu}=\xi^{\rho} D_{\rho} B_{\mu}+D_{\mu} \xi^{\rho} B_{\rho}+\alpha e_{\mu}^{a} \partial_{\theta} \xi^{\rho} \omega_{\rho a} .
\end{aligned}
$$

It remains the question whether actions can be constructed that are manifestly invariant under these transformations. To analyze this let us start with an action constructed from a scalar Lagrangian given by

$$
S=\int d^{3} x d \theta e \mathcal{L},
$$

and moreover being invariant under local Virasoro transformations. Put differently, this means that the Lagrangian varies as $\delta_{\xi^{5}} \mathcal{L}=\xi^{5} \partial_{5} \mathcal{L}-2 \mathcal{L} \partial_{5} \xi^{5}$ under $\hat{v}$ (because then it transforms together with the vielbein determinant, whose symmetry variation reads $\delta_{\xi^{5}} e=\xi^{5} \partial_{5} e+3 e \partial_{5} \xi^{5}$, into a total $\theta$-derivative). By use of the $\hat{v}$-covariant derivative given by

$$
D_{\mu} \mathcal{L}=\partial_{\mu} \mathcal{L}-A_{\mu} \partial_{5} \mathcal{L}+2 \mathcal{L} \partial_{5} A_{\mu},
$$

we can then evaluate the variation of the action under gauged diffeomorphisms and find

$$
\begin{aligned}
\delta_{\xi} S & =\int d^{3} x d \theta\left[\left(\xi^{\rho} D_{\rho} e+e D_{\rho} \xi^{\rho}\right) \mathcal{L}+e \xi^{\rho} D_{\rho} \mathcal{L}\right]=\int d^{3} x d \theta D_{\rho}\left(e \xi^{\rho} \mathcal{L}\right) \\
& =\int d^{3} x d \theta\left[\partial_{\rho}\left(e \xi^{\rho} \mathcal{L}\right)-\partial_{5}\left(e \xi^{\rho} A_{\rho} \mathcal{L}\right)\right]=0 .
\end{aligned}
$$


Thus, if one constructs an action from a Lagrangian that transforms as a scalar under gauged diffeomorphisms, then the action is invariant under these gauged diffeomorphisms if and only if it is also invariant under local Virasoro transformations. The latter requirement is satisfied in our theory by construction. Thus it remains to be checked whether the Lagrangian transforms as a scalar. However, using (4.26), (4.28) and $\left[D_{\mu}, D_{\nu}\right] \phi=-2 \phi \partial_{5} F_{\mu \nu}-\partial_{5} \phi F_{\mu \nu}$, one proves that the covariant derivative $D_{\mu} \phi$ transforms under gauged diffeomorphisms as

$$
\delta_{\xi}\left(D_{\mu} \phi\right)=D_{\mu} \xi^{\rho} D_{\rho} \phi+\xi^{\rho} D_{\rho} D_{\mu} \phi-2 \phi \partial_{5} \xi^{\rho} F_{\rho \mu},
$$

i.e. it does not transform like a one-form, but requires an additional piece proportional to $F_{\mu \nu}$, which again shows that corrections have to be added to the transformation rules.

Let us consider the subsector of the theory where we rescale

$$
e_{\mu}^{a} \rightarrow \kappa e_{\mu}^{a}, \quad \varphi \rightarrow \kappa^{-1 / 2} \varphi,
$$

and then take the limit $\kappa \rightarrow 0$. The action then reads

$$
S_{\kappa \rightarrow 0}=\frac{1}{2} \int d^{3} x d \theta\left(-g \varepsilon^{\mu \nu \rho} B_{\mu} F_{\nu \rho}+e g^{\mu \nu} \phi^{-2} \mathcal{D}_{\mu} \varphi \mathcal{D}_{\nu} \varphi\right) .
$$

However, in spite of the fact that the term $\sim e_{\mu}^{a} \partial_{\theta} \xi^{\rho} \omega_{\rho a}$ in (4.28) disappears in this limit and with the additional contribution (4.25) in the $B_{\mu}$ variation, the extra term in (4.32) is canceled, and the kinetic term for $\varphi$ is therefore separately invariant. The Chern-Simons term on the other hand transforms according to (3.2) as

$$
\delta_{\xi} S_{\kappa \rightarrow 0}=-g \int \varepsilon^{\mu \nu \rho} F_{\sigma \mu} F_{\nu \rho} \varphi \partial_{5} \xi^{\sigma}=0,
$$

where we have used that a totally antisymmetric object in four indices vanishes in $D=3$. Thus we have shown the scalar field modification in (4.25) is sufficient in order to restore the spin-2 invariance of this subsector of the theory.

\section{Conclusions and Discussion}

In this paper we have examined the spin-2 symmetries appearing in Kaluza-Klein theories. Via analyzing an $S^{1}$ compactification to $D=3$ we have shown how a spin-2 symmetry is realized in the unbroken phase by constructing the Chern-Simons theory of the affine Poincare algebra. This spin-2 symmetry fits also into a geometrical framework introduced by Wald. The latter associates to any given commutative algebra a consistent multi-graviton theory, and in the present case the algebra is given by the algebra of smooth functions on $S^{1}$. Even though the Chern-Simons description is special to $D=3$, the latter construction is possible in any dimension. Moreover, by constructing the analogous extension of the Poincaré algebra for arbitrary internal manifolds we prove in the appendix that also in these cases the Chern-Simons theories are equivalent to the algebra-valued gravity theories. Thus we have confirmed and refined the proposal made in [29].

In addition, we discussed matter-couplings in the unbroken phase in a formulation, where all degrees of freedom reside in scalar fields, and showed that they admit an 
enhanced infinite-dimensional rigid symmetry, which contains the Virasoro algebra and an affine extension of the Ehlers group.

Upon gauging part of the global symmetries we constructed the broken phase, which adds a Chern-Simons term for the KK vectors and an explicit mass term for the spin-2 fields. The latter theory was shown to be on-shell equivalent to the Yang-Mills gauged action which one gets by direct KK reduction. Moreover, the KK vectors combined with the metric and the higher spin-2 fields into a Chern-Simons theory for an extended algebra, which features also a central extension for the affine Poincaré subalgebra. The non-abelian gauge transformations for this theory were shown to be on-shell equivalent to the KK transformation in (2.8), up to scalar-dependent terms. This proves that truncating only to the topological fields and disregarding scalar field contributions results in a consistent theory. The latter is in analogy to gauged supergravities in $D=3$, where a truncation to the supergravity multiplet and the compact Chern-Simons gauge fields results in the pure Chern-Simons theories for $A d S$-supergroups constructed in [32]. Similarly, in 33. couplings of an infinite tower of higher-spin fields to gravity have been constructed as Chern-Simons theories for higher-spin algebras, and the present work contains the analogue for an infinite tower of spin-2 fields.

Based on the algebra (4.4) we were able to analyze how the gauging deforms the spin-2 symmetries for the topological fields. But, we can no longer expect this algebra to be realized as a proper gauge symmetry also on the matter fields, since here even ordinary diffeomorphisms have no direct interpretation as Poincaré gauge transformations. We have seen that also scalar-dependent modifications of the spin-2 transformations are required and have shown explicitly how the spin-2 invariance of a subsector (4.34) of the theory is restored. However, we did not analyze in detail which scalar-dependent deformations guarantee the invariance of the full theory containing a spin-2 mass term (whose form can be found in [21]), but just observed that the existence of such a deformation is ensured by the on-shell equivalence to the original KK theory. We will leave a more exhaustive analysis of these deformations, i.e. a complete answer to question (iv) raised in the introduction, for future work. The latter will also be necessary in order to consider more general internal manifolds [34].

Finally let us comment on possible generalizations. Since our original motivation was given by the AdS/CFT correspondence, we would like to analyze KK theories on $A d S_{3}$. For this, naively one would first replace the Poincaré algebra in (4.4) by the $A d S_{3^{-}}$ isometry group $S O(2,2)$. But for non-vanishing cosmological constant $\lambda$ one would get the additional commutator $\left[P_{a}, P_{b}\right] \sim \lambda J_{a b}$, which in turn would render the algebra (4.4) with its non-standard product between $\hat{v}$ and the Kac-Moody algebra inconsistent, as its consistency relied heavily on the nilpotency of the $P_{a}^{m}$. That this algebra would be inconsistent should not be a surprise, because it would correspond to a compactification on $A d S_{3} \times S^{1}$, which is not a solution of four-dimensional AdS-gravity. Accordingly, the naive $S^{1}$-reduction of AdS-gravity in $D=4$ would result in a cosmological constant term in $D=3$ containing an explicit dilaton factor $\phi$, which therefore could clearly not be covered by a Chern-Simons description. On the other hand, for compactifications on, say, $A d S_{3} \times S^{3}$, which are solutions of the higher-dimensional gravity theories, one would expect the appearance of consistent algebras like (4.4), containing an affine extension of $S O(2,2)$.

Moreover, it would be interesting to extend the analysis to the compactification of 
supergravity theories 35]. This would require an extension of (4.4) to a super-Kac-Moody algebra, whose Chern-Simons theory would then describe a supergravity with an infinite number of gravitinos, i.e. possessing an infinite number of supercharges $(N=\infty)$. A resolution of the apparent conflict with the no-go theorem restricting the number of real supercharges to be less or equal to 32 , is given by the observation that upon coupling to propagating matter these theories need not to admit a phase where the whole supersymmetry is unbroken (see also the discussion in [16]).

\section{Acknowledgments}

I am greatly indebted to Henning Samtleben for stimulating discussions at every stage of this project and for carefully reading the manuscript. Moreover, I would like to thank Christian Becker, Jens Fjelstad and Mattias Wohlfarth for useful conversations. This work is supported by the EU contracts MRTN-CT-2004-503369 and MRTN-CT-2004512194, the DFG grant SA 1336/1-1 and DAAD - The German Academic Exchange Service.

\section{A Appendix: Spin-2 theory for arbitrary internal manifold}

In the main text we have shown that the Chern-Simons gauge theory of the affine Poincaré algebra describes a consistent gravity-spin-2 coupling. This is on the other hand also equivalent to Wald's algebra-valued generalization of the Einstein Hilbert action, where the algebra is given by the algebra of smooth functions on $S^{1}$. We are going to show that this picture generalizes to the case of an arbitrary internal manifold.

Let $M$ be an arbitrary compact Riemannian manifold and $\left\{e_{m}\right\}$ a complete set of spherical harmonics (where generically $m$ now denotes a multi-index), which we also take as a basis for the algebra of smooth functions on $M$. The infinite-dimensional extension of the Poincaré algebra is no longer given by a Kac-Moody algebra, but instead is spanned by generators $P_{a}^{n}=P_{a} \otimes e_{n}$ and $J_{a}^{n}=J_{a} \otimes e_{n}$, which satisfy the Lie algebra (compare the algebra in [36])

$$
\left[P_{a}^{m}, P_{b}^{n}\right]=0, \quad\left[J_{a}^{m}, J_{b}^{n}\right]=\varepsilon_{a b c} J^{c} \otimes\left(e_{m} \cdot e_{n}\right), \quad\left[J_{a}^{m}, P_{b}^{n}\right]=\varepsilon_{a b c} P^{c} \otimes\left(e_{m} \cdot e_{n}\right) .
$$

Here - denotes ordinary multiplication of functions. Note, that this algebra reduces for the case $M=S^{1}$ to the Kac-Moody algebra $\hat{i s o(1,2)}$ in (2.11). There exists also an inner product on the space of functions, which is given by

$$
\left(e_{m}, e_{n}\right)=\int_{M} \mathrm{dvol}_{M} e_{m} e_{n},
$$

such that a non-degenerate quadratic form on (A.1) exists:

$$
\left\langle P_{a}^{m}, J_{b}^{n}\right\rangle=\eta_{a b}\left(e_{m}, e_{n}\right)
$$


In complete analogy to sec. 3 a Chern-Simons theory can then be defined, whose equations of motion read

$$
\begin{array}{r}
\partial_{\mu} e_{\nu}^{a(n)}-\partial_{\nu} e_{\mu}^{a(n)}+a_{m k}^{n} \varepsilon^{a b c}\left(e_{\mu b}^{(m)} \omega_{\nu c}^{(k)}+\omega_{\mu b}^{(m)} e_{\nu c}^{(k)}\right)=0, \\
\partial_{\mu} \omega_{\nu}^{a(n)}-\partial_{\nu} \omega_{\mu}^{a(n)}+a_{m k}^{n} \varepsilon^{a b c} \omega_{\mu b}^{(m)} \omega_{\nu c}^{(k)}=0,
\end{array}
$$

while they are invariant under

$$
\delta e_{\mu}^{a(n)}=\partial_{\mu} \rho^{a(n)}+a_{m k}^{n}\left(\varepsilon^{a b c} e_{\mu b}^{(m)} \tau_{c}^{(k)}+\varepsilon^{a b c} \omega_{\mu b}^{(m)} \rho_{c}^{(k)}\right),
$$

where $a_{m k}^{n}$ defines the algebra structure with respect to the basis $\left\{e_{m}\right\}$. If one defines the transformation parameter to be

$$
\rho^{a(n)}=a_{m k}^{n} \xi^{\mu(m)} e_{\mu}^{a(k)}, \quad \tau^{a(n)}=a_{m k}^{n} \xi^{\mu(m)} \omega_{\mu}^{a(k)}
$$

one can show using the equations of motion and the associativity (3.24) of the algebra, that

$$
\delta e_{\mu}^{a(n)}=a_{m k}^{n}\left(\xi^{\rho(m)} \partial_{\rho} e_{\mu}^{a(k)}+\partial_{\mu} \xi^{\rho(m)} e_{\rho}^{a(k)}\right) .
$$

For the algebra-valued metric defined by $g_{\mu \nu}^{n}=a_{m k}^{n} e_{\mu}^{a(m)} e_{\nu a}^{(k)}$ this implies

$$
\begin{aligned}
\delta_{\xi} g_{\mu \nu}^{n} & =\partial_{\mu} \xi^{\rho(l)} g_{\rho \nu l}{ }^{n}+\partial_{\nu} \xi^{\rho(l)} g_{\rho \mu l}{ }^{n}+\xi^{\rho(l)} \partial_{\rho} g_{\mu \nu l}{ }^{n} \\
& =\nabla_{\mu} \xi_{\nu}^{n}+\nabla_{\nu} \xi_{\mu}^{n}
\end{aligned}
$$

where again (3.24) has been used. Altogether, the gauge transformations of the ChernSimons theory for (A.1) coincide with the algebra-diffeomorphisms. Thus we have shown that also for arbitrary internal manifolds the Chern-Simons description based on the algebra (A.1) is equivalent to Wald's algebra-valued multi-graviton theory.

\section{References}

[1] J. M. Maldacena, "The large N limit of superconformal field theories and supergravity," Adv. Theor. Math. Phys. 2 (1998) 231 [Int. J. Theor. Phys. 38 (1999) 1113] arXiv:hep-th/9711200.

[2] E. Witten, "Anti-de Sitter space and holography," Adv. Theor. Math. Phys. 2 (1998) 253 arXiv:hep-th/9802150.

[3] S. S. Gubser, I. R. Klebanov and A. M. Polyakov, "Gauge theory correlators from non-critical string theory," Phys. Lett. B 428 (1998) 105 arXiv:hep-th/9802109.

[4] H. Nicolai and H. Samtleben, "Chern-Simons vs. Yang-Mills gaugings in three dimensions," Nucl. Phys. B 668 (2003) 167 arXiv:hep-th/0303213.

[5] H. Nicolai and H. Samtleben, "Kaluza-Klein supergravity on AdS(3) x S(3)," JHEP 0309 (2003) 036 arXiv:hep-th/0306202. 
[6] D. Sorokin, "Introduction to the classical theory of higher spins," AIP Conf. Proc. 767 (2005) 172 arXiv:hep-th/0405069.

[7] M. Fierz and W. Pauli, "On Relativistic Wave Equations For Particles Of Arbitrary Spin In An Electromagnetic Field," Proc. Roy. Soc. Lond. A 173 (1939) 211.

[8] C. Aragone and S. Deser, "Consistency Problems Of Spin-2 Gravity Coupling," Nuovo Cim. B 57 (1980) 33.

[9] N. Boulanger, T. Damour, L. Gualtieri and M. Henneaux, "Inconsistency of interacting, multigraviton theories," Nucl. Phys. B 597 (2001) 127 arXiv:hep-th/0007220.

[10] A. Hindawi, B. A. Ovrut and D. Waldram, "Consistent Spin-Two Coupling and Quadratic Gravitation," Phys. Rev. D 53 (1996) 5583 arXiv:hep-th/9509142.

[11] C. Cutler and R. M. Wald, "A New Type Of Gauge Invariance For A Collection Of Massless Spin-2 Fields. 1. Existence And Uniqueness," Class. Quant. Grav. 4 (1987) 1267.

[12] R. M. Wald, "A New Type Of Gauge Invariance For A Collection Of Massless Spin-2 Fields. 2. Geometrical Interpretation," Class. Quant. Grav. 4 (1987) 1279.

[13] D. G. Boulware and S. Deser, "Can Gravitation Have A Finite Range?," Phys. Rev. D 6 (1972) 3368.

[14] I. L. Buchbinder, D. M. Gitman, V. A. Krykhtin and V. D. Pershin, "Equations of motion for massive spin 2 field coupled to gravity," Nucl. Phys. B 584 (2000) 615 arXiv:hep-th/9910188.

[15] I. L. Buchbinder, D. M. Gitman and V. D. Pershin, "Causality of massive spin 2 field in external gravity," Phys. Lett. B 492 (2000) 161 arXiv:hep-th/0006144.

[16] O. Hohm and H. Samtleben, "Effective actions for massive Kaluza-Klein states on $\operatorname{AdS}(3)$ x S**3 x S**3," JHEP 0505 (2005) 027 arXiv:hep-th/0503088.

[17] H. Nicolai and H. Samtleben, "Maximal gauged supergravity in three dimensions," Phys. Rev. Lett. 86 (2001) 1686 arXiv:hep-th/0010076.

[18] H. Nicolai and H. Samtleben, "Compact and noncompact gauged maximal supergravities in three dimensions," JHEP 0104 (2001) 022 arXiv:hep-th/0103032.

[19] B. de Wit, I. Herger and H. Samtleben, "Gauged locally supersymmetric D = 3 nonlinear sigma models," Nucl. Phys. B 671 (2003) 175 arXiv:hep-th/0307006.

[20] L. Dolan and M. J. Duff, "Kac-Moody Symmetries Of Kaluza-Klein Theories," Phys. Rev. Lett. 52 (1984) 14.

[21] Y. M. Cho and S. W. Zoh, "Explicit construction of massive spin two fields in Kaluza-Klein theory," Phys. Rev. D 46 (1992) 2290.

[22] Y. M. Cho and S. W. Zoh, "Virasoro invariance and theory of internal string," Phys. Rev. D 46 (1992) 3483. 
[23] Y. M. Cho, "Effective field theory of internal string," Phys. Rev. D 49, 1007 (1994).

[24] C. S. Aulakh and D. Sahdev, "The Infinite Dimensional Gauge Structure Of KaluzaKlein Theories. 1. D = 1+4," Phys. Lett. B 164 (1985) 293.

[25] C. S. Aulakh, "Of Towers And Ladders: Infinite Parameter Symmetries In KaluzaKlein Theories," Phys. Lett. B 146 (1984) 33.

[26] P. Goddard and D. I. Olive, "Kac-Moody And Virasoro Algebras In Relation To Quantum Physics," Int. J. Mod. Phys. A 1 (1986) 303.

[27] E. Witten, “(2+1)-Dimensional Gravity As An Exactly Soluble System," Nucl. Phys. B 311 (1988) 46.

[28] M. J. Duff, C. N. Pope and K. S. Stelle, "Consistent Interacting Massive Spin-2 Requires An Infinity Of States," Phys. Lett. B 223 (1989) 386.

[29] M. Reuter, "Consistent Interaction For Infinitely Many Massless Spin Two Fields By Dimensional Reduction," Phys. Lett. B 205 (1988) 511.

[30] J. Ehlers, "Konstruktion und Charakterisierungen von Lösungen der Einsteinschen Gravitationsgleichungen," Dissertation, Hamburg (1957).

[31] O. Hohm and J. Louis, "Spontaneous $\mathrm{N}=2 \rightarrow \mathrm{N}=1$ supergravity breaking in three dimensions," Class. Quant. Grav. 21 (2004) 4607 arXiv:hep-th/0403128.

[32] A. Achucarro and P. K. Townsend, "A Chern-Simons Action For Three-Dimensional Anti-De Sitter Supergravity Theories," Phys. Lett. B 180 (1986) 89.

[33] M. P. Blencowe, "A Consistent Interacting Massless Higher Spin Field Theory In D $=(2+1)$, , Class. Quant. Grav. 6 (1989) 443.

[34] C. S. Aulakh and D. Sahdev, "Infinite Dimensional Gauge Structure Of Kaluza-Klein Theories. 2. D > 5," Phys. Lett. B 173 (1986) 241.

[35] L. Dolan, "Symmetries Of Massive Fields In Kaluza-Klein Supergravity," Phys. Rev. D 30 (1984) 2474.

[36] N. Boulanger, M. Henneaux and P. van Nieuwenhuizen, "Conformal (super)gravities with several gravitons," JHEP 0201 (2002) 035 arXiv:hep-th/0201023. 